\title{
GEOMETRIC CALCULUS: DIFFERENTIAL FORMS AND INTEGRATION
}


Gottfried Wilhelm Leibniz's notion of infinitesimal analysis, culminating in the development of the theory of differentials, targets the development of a genuine type of "geometric calculus" by algebraic means, one based not on artificial choices of coordinates and other subjective conventions, but pertaining to the geometric objects themselves, as an articulation of an extension process from the infinitesimal to the global. The notion of a geometric object is conceived in terms of the spectrum of a commutative ring or, an algebra over a field, like the real or complex numbers. The idea of a spectrum, originating from physics, refers to what can be observed through this ring by evaluating it into a field of measurement scales. In this sense, the ring or, algebra, on which a differentiation procedure may be applied, as pertaining to an infinitesimal type of extension, is qualified as an algebraic structure whose elements are observables.

In this line of thought, the ideal theory of rings, conceived first by Richard Dedekind, provides the necessary additional conceptual and technical metaphorical means for a precise rendering of Leibniz's ideas in modern terms.

A non-empty subset $\mathcal{I}$ of a commutative ring $\mathcal{A}$ is called an ideal if the following properties hold:

i $\mathcal{I}$ is an additive subgroup of $\mathcal{A}$, i.e. for every $x, y \in \mathcal{I}$, we have $x-y \in \mathcal{I}$;

ii $\quad \mathcal{I}$ is stable with respect to multiplication with elements of the ring $\mathcal{A}$, viz., for every $x \in \mathcal{I}$ and for every $y \in \mathcal{A}$, the product $x y$ is being absorbed in $\mathcal{I}$, viz. $x y \in \mathcal{I}$.

It is clear that for every ring $\mathcal{A}$, the subsets 0 and $\mathcal{A}$ itself are ideals of $\mathcal{A}$, the trivial ideals. Moreover, if $\mathcal{I}$ contains an invertible element of $\mathcal{A}$, then $\mathcal{I}=\mathcal{A}$. Then, the only ideals of a field are 0 and the field itself. Let us now consider a homomorphism of rings $u: \mathcal{A} \rightarrow \mathcal{B}$, to be physically thought of as a measurement procedure of a ring of observables $\mathcal{A}$, by a ring of measurement scales $\mathcal{B}$. We recall that the kernel of $u$ is defined as follows:

$$
\operatorname{Ker}(u):=\{a \in \mathcal{A} \mid u(a)=0\}=u^{-1}(0)
$$

Then $\operatorname{Ker}(u)$ is clearly an ideal of $\mathcal{A}$. The kernel of the homomorphism $u$ depicts the set of observables in $\mathcal{A}$, whose evaluation is the neutral 
additive element of the rings of scales $B$. Hence, the ideal $\operatorname{Ker}(u)$ of the ring of observables $\mathcal{A}$, can be interpreted geometrically as a schematization perspective, with respect to which the measurement procedure $u: \mathcal{A} \rightarrow \mathcal{B}$ is effectuated. Obviously, the set of all observables in $\mathcal{A}$ constituting a perspective, identified as the ideal $\operatorname{Ker}(u)$ of $\mathcal{A}$, in relation to a measurement procedure $u: \mathcal{A} \rightarrow \mathcal{B}$, are being evaluated to the 0 of the ring of scales $\mathcal{B}$.

Epigrammatically, we may say that a geometric schematization perspective related with a measurement procedure of a ring of observables, constitutes the kernel of its evaluation at a ring of scales identified with the inverse image of the additive neutral element in $\mathcal{B}$, which is the zero scale.

This basic conceptual point can be further clarified by introducing the notion of a quotient ring. More concretely, if we have at our disposal a ring $\mathcal{A}$ and an arbitrary ideal $\mathcal{I} \subseteq \mathcal{A}$, then, we can define the quotient ring $\mathcal{A} / \mathcal{I}$. The essence of this construction amounts to declaring equal to zero everything that is in $\mathcal{I}$. We can transform this idea into something precise by remarking that, if every element of $\mathcal{I}$ has to be considered as zero, then two elements $x, y$ of $\mathcal{A}$ whose difference $x-y$ is in the ideal $\mathcal{I}$ have to be considered as equal.

This procedure produces a new ring, the pertinent quotient ring as follows: We define the following equivalence relation in $\mathcal{A}: x \vee y$ if and only if $x-y \in \mathcal{I}$, where $x, y \in \mathcal{A}$. The key feature of the equivalence relation defined above, is that it is compatible with the ringtheoretic operations on $\mathcal{A}$, or else, it is being preserved by addition and multiplication in $\mathcal{A}$, formally, if $x \diamond x^{\prime}$ and $y \diamond y^{\prime}$, then $x+y$ $\diamond x^{\prime}+y^{\prime}$ and $x y \quad \diamond \quad x^{\prime} y^{\prime}$. Therefore, we can legitimately define two operations on the quotient set $\mathcal{A} / \diamond:=\mathcal{A} / \mathcal{I}$, and hence, $\mathcal{A} / \diamond$ becomes a ring with respect to these operations, that is, the quotient ring $\mathcal{A} / \mathcal{I}$.

In order to translate the information encoded into the ring $\mathcal{A}$, into the quotient ring $\mathcal{A} / \mathcal{I}$ we employ a morphism of rings $\mathcal{A} \rightarrow \mathcal{A} / \mathcal{I}$. In fact, the canonical morphism $l: \mathcal{A} \rightarrow \mathcal{A} / \mathcal{I}$ given by $a \mapsto[a]:=a+\mathcal{I}$ is a surjective morphism of rings, the canonical epimorphism. Thus, we finally have:

$$
\operatorname{Ker}(\imath)=\mathcal{I}
$$

The above clarifies the essential idea behind the construction of the quotient ring, of declaring equal to zero everything that is in $\mathcal{I}$. Put 
simply, when we change the ring from $\mathcal{A}$ to $\mathcal{A} / \mathcal{I}$ by means of the canonical epimorphism $l$, then everything in $\mathcal{I}$ goes to zero.

Thus, in relation to the intended interpretation, we form the following conclusion: a geometric schematization perspective related with a measurement procedure of a ring of observables $\mathcal{A}$, identified as an ideal $\mathcal{I}$ of $\mathcal{A}$, constitutes the kernel of its evaluation at a ring of scales, identified, in turn, as the quotient ring $\mathcal{A} / \mathcal{I}$.

From then on, based on the information encoded in the quotient ring $\mathcal{A} / \mathcal{I}$ we can easily obtain the following classifications: an ideal $\mathcal{I}$ is maximal if and only if $\mathcal{A} / \mathcal{I}$ is a field, whereas an ideal $\mathcal{I}$ is prime if and only if $\mathcal{A} / \mathcal{I}$ is an integral domain, or equivalently, a ring without divisors of zero. Obviously, every maximal ideal is a prime ideal.

Intuitively, a maximal ideal is an ideal which is second only to the entire ring. A prime ideal can be conceived as a measure of the complexity of a ring. This is based on the observation that if a ring $\mathcal{B}$ is a field, then there is only one prime ideal, namely the zero ideal, since it is the only ideal besides $\mathcal{B}$, and moreover, it is prime since $\mathcal{B}$ is an integral domain. Thus, prefixing the ring of measurement scales to a field, as in classical physical theories, where the field is that of the real numbers, we assume a zero complexity of scales. A scale of zero complexity is unable to register a stable irreducible process, or a process of indistinguishability between the observed and the means of observation, and hence, it coordinatizes the geometric spectral point-states, corresponding to maximal ideals in the ring of observables, in terms of numbers. Hence, the state-manifolds constructs of classical theories are geometric spaces built on the assumption of zero complexity of measurement scales, and consequently, constitute a kind of an arithmetic continuum, which, incorporates the hypothesis of a pre-assigned demarcation boundary between the observed and the observational means.

If we consider instead the ring of the integers $\mathcal{Z}$ as a ring of measurement scales, the prime ideals are the maximal ideals $p \mathcal{Z}$, where $p$ is a prime number, as well as the zero ideal. Then, it is not a coincidence that prime integer measurement scales coordinatize stable and not further reducible spectral perspectives, represented geometrically as pure states. More generally, the notion of a prime ideal in an abstract ring of measurement amounts to a stable, and not further reducible spectral perspective, playing exactly the role of a measure of irreducible complexity.

In this line of reasoning, any ideal in a ring expresses a stable perspective geometrically, capable of being decomposed or factorized into perspectives of irreducible complexity enunciated by prime ideals. Equally from this viewpoint, the algebraic theory of factorization of rings 
into prime or even primary ideals deserves special attention. We point out that the notion of a primary ideal generalizes that of a prime ideal, in the sense that it is characterized by a quotient ring that is allowed to have zero divisors, but constrains them to be strictly nilpotent. Moreover, both primary and prime ideals, in contradistinction to maximal ideals, are characterized by a covariance property under homomorphisms of rings, meaning that they have inverse images that are still primary or prime, hence, they behave well under base ring change.

The duality between commutative algebras of observables and geometric state-spaces, is based primarily on the idea that evaluating an observable $f$ at a state $x$ is the same as evaluating $x$ at the observable $f$ :

$$
x(f)=f(x)
$$

More precisely, if we consider the simplest possible case, where, $\mathcal{A}$ is the $\mathcal{F}$-algebra of functions on a set $X$ with values in a field $F$, any element $x \in X$ defines a morphism $\mathcal{A} \rightarrow \mathcal{F}$ by assigning to a function $f$ its value at $x$. The kernel of this morphism is a maximal ideal $\mu$ in $\mathcal{A}$, suggesting in this way that it is possible to recover the set $X$ as the set of maximal ideals in $\mathcal{A}$. In case that, $\mathcal{A}=\mathcal{C}^{\infty}(N)$, the maximal ideal $\mu_{x}$ is the ideal consisting of all smooth functions vanishing at the point $x$ :

$$
\mu_{x}=\left\{f \in \mathcal{C}^{\infty}(N) \mid f(x)=0\right\}
$$

Notice that the definition above, forces a decomposition of the algebra $\mathcal{C}^{\infty}(N)$ into a direct sum of linear vector spaces:

$$
\begin{gathered}
\mathcal{C}^{\infty}(N)=\mathcal{R} \oplus \mu_{x} \\
f=f(x)+(f-f(x))
\end{gathered}
$$

and furthermore, the quotient ring $\mathcal{C}^{\infty}(N) / \mu_{x}$ is isomorphic to the field of real numbers $\mathcal{R}$.

We proceed by defining more concretely the notion of a pure state over a ring of measurement scales. Let us assume that $\mathcal{A}$ is a commutative, unital $\mathcal{F}$-algebra of observables, where $\mathcal{F}$ is an 
arbitrary field, and moreover, let $\mathcal{B} \supset \mathcal{F}$ be a ring without zero divisors (integral domain), interpreted as a ring of measurement scales.

We define a pure state of $\mathcal{A}$ over the ring $\mathcal{B}$ as a surjective morphism of $\mathcal{F}$-algebras $w: \mathcal{A} \rightarrow \mathcal{B}$. The notion of a pure state of $\mathcal{A}$ over $\mathcal{B}$ instantiates a schematizing, stable, and irreducible natural perspective, encoded geometrically, via the scales of $B$, by means of evaluations of observables of $\mathcal{A}$ into $\mathcal{B}$, within the context of a corresponding measurement procedure.

Moreover, we say that two pure states, defined over the rings, $\mathcal{B}_{1}$ and $\mathcal{B}_{2}$ respectively, specified as above, are identical, if there exists an isomorphism of $\mathcal{F}$-algebras $\mathcal{B}_{1} \cong \mathcal{B}_{2}$, effecting an isomorphism between the corresponding measurement scales. Hence, we may define a $\mathcal{B}$-pure state of an $\mathcal{F}$-algebra of observables $\mathcal{A}$, as an equivalence class of surjective morphisms of $\mathcal{F}$-algebras $w: \mathcal{A} \rightarrow \mathcal{B}$, where, the ring $\mathcal{B}$ $\supset \mathcal{F}$ is called the coordinatizing frame of the pure state $w$.

Notice that according to the definition introduced, each pure state of an $\mathcal{F}$-algebra of observables $\mathcal{A}$ may have a different coordinatizing frame, depending upon the integral domain of scales employed for measurement. Thus, the new notion of a geometric state-space, being built from pure states, is a multi-valued one, in the sense that its generalized point-states may be coordinatized by means of different scales, namely scales belonging to different integral domains.

At this stage, it is essential to emphasize for reasons of clarity, that the identification of pure states with generalized points of a multi-valued geometric state space, corresponding to irreducible perspectives of observation or measurement, is precisely established by the existence of a bijective correspondence between $\mathcal{B}$-pure states of an $\mathcal{F}$-algebra of observables $\mathcal{A}$, where, $B \supset \mathcal{F}$ is a coordinatizing ring without zero divisors, and the prime ideals of the algebra $\mathcal{A}$. The bijection can be established by defining the following assignment:

$$
[w: \mathcal{A} \rightarrow \mathcal{B}] \mapsto \operatorname{Ker}(w)
$$

It is easy to verify, if we consider a prime ideal $\mathcal{I}$ of $\mathcal{A}$, that the quotient ring $\mathcal{A} / \mathcal{I}$ must be an integral domain, and that the canonical epimorphism $l: \mathcal{A} \rightarrow \mathcal{A} / \mathcal{I}$ is an $\mathcal{A} / \mathcal{I}$-pure state of the algebra $\mathcal{A}$, such that, $\operatorname{Ker}(i)=\mathcal{I}$.

Thus, we identify the set of all pure states of an $\mathcal{F}$-algebra of observables $\mathcal{A}$ with the set of all prime ideals of $\mathcal{A}$, or equivalently, with the prime spectrum of $\mathcal{A}$, denoted by $P S p e c \mathcal{A}$. This term denotes 
the set of all equivalence classes of stable and irreducible spectral schematization perspectives, coordinatized through the measurement scales of integral domains, by means of evaluations of observables of $\mathcal{A}$ into $\mathcal{B}$.

The definition of $P \operatorname{Spec} \mathcal{A}$ allows us to think of an observable $f$ $\in \mathcal{A}$, intuitively, as a function on $P \operatorname{Spec} \mathcal{A}$, in the sense that we have values $f(\mathcal{I})$, defined for each state $\mathcal{I} \in P \operatorname{Ppec} \mathcal{A}$. However, these functions have the property that the space where their values live varies depending on the state where the evaluating is being performed.

Therefore, we may summarize briefly the conceptual shift involved in the definition of a pure state by comparison with the classical definition as follows: In the case of the algebra of observables $\mathcal{C}^{\infty}(N)$, viz., an $\mathcal{R}$-algebra of smooth real-valued functions on a compact real differential manifold $N$, the value of a function $f \in \mathcal{C}^{\infty}(N)$ at a state $\chi$, corresponding to a maximal ideal $\mu_{\chi}$ of $\mathcal{C}^{\infty}(N)$ lives in the quotient ring $\mathcal{C}^{\infty}(N) / \mu_{\chi}$, and all of these quotient rings can be canonically identified with the field of real numbers $\mathcal{R}$. In contradistinction, the notion of a generalized pure state introduced here stands opposed to the absolute representability principle of the classical theory over the coordinatizing field of real numbers. In its own terms, rather, it allows the geometric representation of stable and irreducible spectral perspectives, in terms of generalized points of a multi-valued geometric state space, and the evaluation of observables at these points. This is achieved by relativizing representability over a multitude of measurement scales, belonging to different coordinatizing rings without zero divisors, giving rise eventually to the above multi-valued geometric state space, constructed as the prime spectrum of the corresponding ring of observables.

It is particularly elucidating to clarify the peculiar nature of this multivalued geometric state space. For this purpose, we consider an observable that vanishes at all states. We will show, by using a simple example, that such an observable is not necessarily zero. Consider the $\mathcal{F}$-algebra of dual numbers $\mathcal{F}[\varepsilon] /(\varepsilon)^{2}$ over $\mathcal{F}$.

Formally, the elements of the algebra $\mathcal{F}[\varepsilon] /(\varepsilon)^{2}$ are constituted by the linear combinations $a+b \varepsilon$, where $a, b \in \mathcal{F}$ and $\varepsilon$ is a formal symbol, enunciated as follows: The addition is given by adding 
coefficient-wise, whereas the multiplication is given by applying $\varepsilon^{2}=0$, thus algebraically rendering $\varepsilon$ an infinitesimal unit. Hence, we have that $\varepsilon \neq 0$, but $\varepsilon^{2}=0$. Then, the prime spectrum of this algebra, $\operatorname{PSpec}\left(\mathcal{F}[\varepsilon] /\left(\varepsilon^{2}\right)\right)$ consists of a single generalized point-state, corresponding to the unique prime ideal $(\varepsilon)$.

The $\mathcal{F}$-algebra of dual numbers is the smallest ring with nilpotent elements. Hence, we may consider an observable $\varepsilon$ on $\operatorname{PSpec}\left(\mathcal{F}[\varepsilon] /\left(\varepsilon^{2}\right)\right)$ that is nilpotent, namely the observable $\varepsilon$ itself is not the zero function, but its square $\varepsilon^{2}$ is the zero function. Note that in this example, the fact that $\varepsilon^{2}=0$ means that the observable $\varepsilon$ takes the value 0 at every (the) state of $\operatorname{PSpec}\left(\mathcal{F}[\varepsilon] /\left(\varepsilon^{2}\right)\right)$, but $\varepsilon$ is not considered to be the zero function.

Generalizing, we state that any observable vanishing at all states might not be zero, but some power of it will be zero. In conclusion, observables on the multi-valued geometric space $P \operatorname{Spec} \mathcal{A}$ will not be entirely determined by their values at states, and thus, we no longer distinguish observables based on their values at states. Hence, observables on $P \operatorname{Spec} \mathcal{A}$ will have values at states, but are not going to be determined by those values, instead, they will be entirely determined by their germs. In order to explain the situation clearly, it is necessary to endow $P S p e c \mathcal{A}$ with a sheaf structure, that intuitively constitutes an encoding of observables which is local and global in a compatible way, as we shall see as we go on.

At this point, we must study the generation of the $\mathcal{F}$-algebra of dual numbers $\mathcal{F}[\varepsilon] /(\varepsilon)^{2}$ from $\mathcal{F}$ in more detail. Since this extension pertains to any algebra of observables defined over $\mathcal{F}$, as an infinitesimal extension, it is important to examine the general case, where for simplicity the scalars can be identified with the real numbers, meaning that $\mathcal{F} \equiv \mathbb{R}$, in this case.

In general algebraic terms, the process of extending the observables of an $\mathbb{R}$-algebra $A$ is described by means of a fibration, defined as an injective homomorphism of $\mathbb{R}$-algebras $l: A \infty B$. Thus, the $\mathbb{R}$-algebra $B$ is considered as a module over the algebra $A$, analogously to a vector space over a field. A section of the fibration $l: A \infty B$, is represented by a homomorphism of $\mathbb{R}$-algebras $s: B \rightarrow A$ such that $l \circ S=i d_{B}$.

The fundamental extension of observables of the $\mathbb{R}$-algebra $A$ is obtained by tensoring $A$ with itself over the subalgebra of the base 
field, that is $l: A \infty A \otimes_{\mathbb{R}} A$. Trivial cases of extensions, in fact isomorphic to $A$, induced by the fundamental one, are obtained by tensoring $A$ with $\mathbb{R}$ from both sides, that is $l_{1}: A \infty A \otimes_{\mathbb{R}} \mathbb{R}$, $l_{2}: A \infty \mathbb{R} \otimes_{\mathbb{R}} A$.

The basic idea of Leibniz in conceptualizing infinitesimal analysis as a geometric calculus, which was further elaborated by Riemann in relation to the development of differential geometry, is that it should be thought of in terms of the extension from the infinitesimal to the global. For this purpose, we consider the extension of the algebra of observables $A$ by infinitesimal quantities, defined as the fibration:

$$
\begin{aligned}
d_{*} & : A \infty A \oplus M \cdot \varepsilon \\
f & \mapsto f+d(f) \cdot \varepsilon
\end{aligned}
$$

where $d(f)=: d f$ is understood as the infinitesimal part of the extended observable, and $\varepsilon$ denotes the infinitesimal unit obeying $\varepsilon^{2}=0$. The algebra of infinitesimally extended observables $A \oplus M \cdot \varepsilon$ is called the algebra of dual numbers over $A$ with coefficients in the $A$ module $M$. It is immediately evident that the algebra $A \oplus M \cdot \varepsilon$, as an Abelian group is just the direct sum $A \oplus M$, whereas the multiplication is defined as follows:

$$
(f+d f \cdot \varepsilon) \bullet\left(f^{\prime}+d f^{\prime} \cdot \varepsilon\right)=f \cdot f^{\prime}+\left(f \cdot d f^{\prime}+f^{\prime} \cdot d f\right) \cdot \varepsilon
$$

It is also required that the composition of the augmentation $A \oplus M \cdot \dot{U} \rightarrow A$, with $d_{*}$ is the identity.

Equivalently, the above fibration defined by the injective homomorphism of algebras $d_{*}: A \infty A \oplus M \cdot \varepsilon$, can be reformulated as a derivation, that is in terms of an additive $\mathbb{R}$-linear morphism:

$$
\begin{gathered}
d: A \rightarrow M, \\
f \mapsto d f
\end{gathered}
$$

that satisfies the Leibniz rule:

$$
d(f \cdot g)=f \cdot d g+g \cdot d f
$$

Seen in this light, the Leibniz rule descends from the linearization of the above fibration. Since the formal symbols of differentials $\{d f, f \in A\}$, are 
reserved for the universal derivation, the $A$-module $M$ is identified as the free $A$-module $\Omega:=\Omega^{1}(A)$ of differential 1 -forms generated by these formal symbols, modulo the Leibniz rule, where the scalars of the distinguished subalgebra $\mathbb{R}$, that is the real numbers, are treated as constants.

The fundamental insight of Erich Kähler in this algebraic setting consists in the realization that the free $A$-module $\Omega$ can be constructed explicitly through the tensor product self-extension of $A$, that is $l: A \infty A \otimes_{\mathbb{R}} A$ by considering the homomorphism:

$$
\begin{gathered}
\mu: A \otimes_{\mathbb{R}} A \rightarrow A \\
\sum_{i} f_{i} \otimes g_{i} \mapsto \sum_{i} f_{i} \cdot g_{i}
\end{gathered}
$$

Then, by taking the kernel of this homomorphism of algebras, that is, the ideal:

$$
I=\operatorname{Ker} \mu=\left\{\theta \in A \otimes_{\mathbb{R}} A: \mu(\theta)=0\right\} \subset A \otimes_{\mathbb{R}} A
$$

we obtain the following: The homomorphism of $A$-modules

$$
\begin{gathered}
\Sigma: \Omega \rightarrow \frac{I}{I^{2}} \quad(10) \\
d f \mapsto 1 \otimes f-f \otimes 1
\end{gathered}
$$

is an isomorphism.

Thus, the free $A$-module $\Omega$ of 1 -forms is isomorphic with the free $A$-module $\frac{I}{I^{2}}$ of Kähler differentials of the algebra of observables $A$ over $\mathbb{R}$, conceived as distinguished ideals within the algebra of infinitesimally extended scalars $A \oplus \Omega \cdot \varepsilon$, according to the following split short exact sequence:

$$
\Omega \longmapsto A \oplus \Omega \cdot \varepsilon \rightarrow A,
$$

equivalently formulated as:

$$
0 \rightarrow \Omega \rightarrow A \otimes_{\mathbb{R}} A \rightarrow A
$$


By dualizing, we obtain the dual $A$-module of $\Omega$, that is, $\Xi:=\operatorname{Hom}(\Omega, A)$. Consequently, we have at our disposal, expressed in terms of infinitesimal extension of the algebra $A$, semantically intertwined with the generation of geometry from the infinitesimal to the global new types of observables related with the incorporation of differentials and their duals, identified as vectors.

If the geometry is generated by a metric, then there is an associated unique dual to a vector, meaning an isomorphism $\tilde{g}$ between the $A$ module $\Omega$ and its dual $A$-module $\Xi=\operatorname{Hom}(\Omega, A)$, that is:

$$
\tilde{g}: \Omega \rightarrow \Xi,(14)
$$

such that:

$$
\begin{aligned}
\tilde{g}: \Omega & \cong \Xi, \quad(15) \\
d f \mapsto v_{f} & :=\tilde{g}(d f) .
\end{aligned}
$$

Equivalently, a metric $g$ stands for an $\mathbb{R}$-valued symmetric bilinear form on $\Omega$, that is $g: \Omega \times \Omega \rightarrow \mathbb{R}$, yielding an invertible $\mathbb{R}$-linear morphism $\tilde{g}: \Omega \rightarrow \Xi$. Notice that for $d f, d h \in \Omega$, a symmetric bilinear form $g$ acts, via $\tilde{g}$, on $d f$ to give an element of the dual, $\tilde{g}(d f) \in \Xi$, which then acts on $d h$ to give $(\tilde{g}(d f))(d h)=(\tilde{g}(d h))(d f)$, or equivalently, $v_{f}(d h)=v_{h}(d f) \in \mathbb{R}$. Also note that the invertibility of $\tilde{g}$ amounts to the property of non-degeneracy of $g$, meaning that for each $d f \in \Omega$, there exists $d h \in \Omega$, such that $(\tilde{g}(d f))(d h)=v_{f}(d h) \neq 0$.

\section{LINEAL EXTENSION: MULTIPLICATION OF DIRECTED LINE SEGMENTS}

The geometric essence of Leibniz's infinitesimal analysis, independently of any notion of absolute pre-existing space in the original sense of a geometric calculus, can be enunciated utilizing the metaphora involved in the development of Hermann Grassmann's theory of lineal extension. The latter emerged out of Grassmann's struggle to establish a universal apparatus for research in geometry by algebraic means, based on the notion of extension. The latter is implemented as a lineal process of unfolding geometric observables in a series of hierarchically organized, and nested layers. This process is expressed in terms of the conceptually inverse geometric actions of joining and separating, involving the ascent 
and descent respectively, from one layer to another layer. The crucial aspect of this theory is that the algebraic encapsulation of lineal extension necessarily requires the operation of multiplication, i.e. the product operation specifying the composition type of geometric observables from layer to layer.

In Grassmann's conception the process of extension is initiated at the first geometric layer, which is occupied by directed line segments, to be thought of in modern terms, as vectors. It is very important that the existence of a zero geometric layer, identified with the scalars, is also assumed, but only implicitly, and not explicitly, as is the case starting with the first layer. The significance of the above is that lineal extension theory actually constitutes an indirectly self-referential schema for the articulation of the notion of a geometric point through a many-layered metaphora conducted by means of ascending to higher and higher geometric layers, and then descending back. We argue that it is precisely this feature that sheds light on the geometric essence of calculus, in such a way that Grassmann's theory cannot be viewed independently from Leibniz's infinitesimal analysis.

The algebraic operation of multiplication that allows ascending is conceived indirectly in relation to the operation of addition. More specifically, if there is an operation identified as addition, which is operative within a single layer, then any operation satisfying the distributive law in terms of this addition is called multiplication. In this manner, Grassmann introduced the operation of multiplication of directed line segments, called the exterior product, as a means of their composition, according to the satisfaction of the distributive law with respect to the addition of directed line segments.

The exterior product is an associative, multiplicative operation described as follows, when applied at the first geometric layer: It takes two directed line segments $A$ and $B$ at the first layer, and produces a directed area or parallelogram at the second layer. This can be accomplished in two different ways that are not equivalent to each other. Either the parallelogram $A \vee B$ is the product of replicating $B$ a total of $A$ times along the linear extension of $A$, and then concatenating or adding these based replications, or the parallelogram $B \vee A$ is the product of replicating $A$ a total of $B$ times along the linear extension of $B$ and then adding these based replications again.

Consequently, we obtain two oppositely-oriented extension processes from the linear layer to the bilinear or area-bounding layer. They mutually cancel each other in the precise sense that their product is oriented oppositely, meaning that $A \vee B=-B \vee A$. The fact that the multiplication product area of the linear extensions $A$ and $B$ is directed or signed, according to the above, means that this geometric 
product is non-commutative. Unfortunately in the literature, the "join- $\vee$ " composition operation expressed by the exterior product is denoted by the "conjunction- $\wedge$ " sign, whose symbolism is the exact opposite to the one intended by this operation. Keeping in mind this cautious remark, we may switch to the "wedge-notation" used in exterior algebra in order to conform with that convention in the literature, according to which $A \wedge B=-B \wedge A$.

We recapitulate that the multiplicative exterior product of two linear directed segments is non-commutative; it depends on the order of their composition, and this can take place in two possible, oppositelyoriented ways. Henceforth, the orientation of the produced signed area is dependent on the order of composition, and thus it is signed. If we identify the first geometric layer in which the independent linear extensions $A$ and $B$ are located as the first exterior power space, then the layer that the product signed area is located is identified as the second exterior power space. In a totally analogous fashion, the lineal extension process proceeds to higher exterior power spaces using the property of associativity of the exterior product and the property of distributivity with addition.

Analogously to the directed area element $A \wedge B$, the lineal extension process instantiates, at the third geometric layer, the directed volume element $A \wedge B \wedge C$ for three independent linear extensions $A$, $B, C$. Due to the associativity property, this should also be thought of in a temporally ordered way, by replicating the directed area of two of them along the linear extension of the third, and then concatenating to obtain the directed volume element. Note that, due to the antisymmetric property of the exterior product, if two elements at a layer have a common element of a lower layer, then their product is zero. This provides a conceptual understanding of the lineal extension process driven by the application of this multiplicative product. More precisely, it is named exterior because the non-nullity of the product of two extensive geometric magnitudes requires that each one of them is located geometrically to the exterior of the other making them independent.

If we consider a finite dimensional vector space $V$ of dimension $v$ over a field $\mathbb{K}$, then the first layer is identified with the first exterior power vector space $\wedge^{1} V:=V$, the second layer with the second exterior power vector space $\wedge^{2} V$, and so on. The characteristic of this extension 
process is that in the $\mu$ exterior power vector space, where $\mu<v$, we have:

$$
\ldots \wedge v_{i} \wedge \ldots \wedge v_{j} \wedge \ldots=0 \quad \text { if } \quad v_{i}=v_{j} \quad \forall i \neq j
$$

expressing the pertinent alternating property. In each exterior power space layer, addition of exterior products located at this layer is allowed, so the general elements of these power spaces are linear superpositions of products of elements descending to lower layers. Moreover, the vector $v_{1}$, $v_{2}, v_{3}, \ldots v_{\mu}$ are linearly independent in $V$, where $\mu \leq v$, if and only if

$$
v_{1} \wedge v_{2} \wedge \ldots \wedge v_{\mu} \neq 0 \text { in } \wedge^{\mu} V
$$

The exterior product underlying the lineal extension process from layer to layer may be considered as a bilinear map from $\wedge^{\lambda} V \times \wedge^{\mu} V$, where $\lambda, \mu \leq \nu$, to $\wedge^{\lambda+\mu} V$, where $\lambda+\mu \leq n$. Hence, for $\circlearrowright \in \wedge^{\lambda} V$, and $\sigma \in$ $\wedge^{\mu} V$, their exterior product is an element $\circlearrowright \wedge \sigma \in \wedge^{\lambda+\mu} V$, meaning that this is a bilinear operation on $\circlearrowright, \sigma$. As an immediate consequence of the alternating property of the exterior product, we obtain that

$$
\circlearrowright \wedge \sigma=(-1)^{\lambda \mu} \sigma \wedge \circlearrowright
$$

Being endowed with the exterior multiplication product, which is associative and distributive over addition, the direct sum of all exterior power vector spaces forms a non-commutative algebra over $\mathbb{K}$ (we exclude the case that $1=-1$ in $\mathbb{K}$ ):

$$
E(V)=\mathbb{K}+V+\stackrel{2}{\wedge} V+\stackrel{3}{\wedge} V+\ldots
$$

Note that $\operatorname{dim} \wedge^{\mu} V=\frac{v !}{\mu !(v-\mu) !}$, and the direct sum terminates at $\mu=v$. Consequently, the top exterior power is one-dimensional, and $\operatorname{dim} E(V)=2^{v}$.

For example, consider that $V$ is a real vector space of dimension 3 , with basis $e_{1}, e_{2}, e_{3}$. Then, we obtain that 


$$
\begin{gathered}
\wedge^{V}=\mathbb{R} \\
\wedge^{1} V=V=\mathbb{R} e_{1} \oplus \mathbb{R} e_{2} \oplus \mathbb{R} e_{3} \\
\wedge^{2} V=\mathbb{R}\left(e_{1} \wedge e_{2}\right) \oplus \mathbb{R}\left(e_{1} \wedge e_{3}\right) \oplus \mathbb{R}\left(e_{2} \wedge e_{3}\right) \\
\wedge^{2} V=\mathbb{R}\left(e_{1} \wedge e_{2} \wedge e_{3}\right)
\end{gathered}
$$

The exterior algebra $E(V)$ is the direct sum of the above exterior power vector spaces and its dimension is $1+3+3+1=8=2^{3}$.

In the same manner that Grassmann's lineal extension process applies to vectors, it analogously applies to their dual objects, called covectors or linear forms. Moreover, it also applies to a linear transformation $\phi$ of a vector space, where in this case, the top exterior power of $\phi$, i.e. $\wedge^{v} \phi: \wedge^{v} V \rightarrow \wedge^{v} V$, is scalar multiplication with the determinant of $\phi$. Furthermore, the same extension process can be transferred to free modules, and finitely generated locally free modules, over a commutative algebra of observables.

We emphasize that the total exterior algebra of a vector space $V$ is characterized universally by an intrinsic feature that is independent of its constituent layer-by-layer exterior power vector spaces. More precisely, if we consider any $\mathbb{K}$-algebra $Q$ and $a \mathbb{K}$-linear map from $V$ to $Q$, i.e. $l: V \rightarrow Q$, such that the square of the image elements of $V$ in $Q$ is zero, it can always be extended uniquely, up to a unique isomorphism, to an $\mathbb{K}$-algebra map from $E(V)$ to $Q$. This is a universal property, and thus characterizes $E(V)$ uniquely in the fashion of category theory. In other words, every linear map from $V$ to $Q$ having the square zero property in the image, factorizes uniquely through the exterior algebra $E(V)$. This intrinsic feature of $E(V)$ plays a decisive role in what is to follow.

Each exterior power vector space, considered within the exterior algebra $E(V)$, delineates a homogenous part of this total algebra, in the sense that its elements instantiate the homogenous elements of the degree specified by the specific exterior power. The homogenous elements of a degree injected into the total exterior algebra may be thought of as the rays corresponding to the pertinent exterior power in the lineal extension process forming this total graded algebra. Reciprocally, the whole process descends back to the level of directed linear extensions, where we should recall that the scalars occupying the zero layer are only implicitly assumed. 
Conceptually, we may think of a directed linear extension in temporal terms, in the sense of a provisional order of actions referring to the replication of another independent linear extension along it, according to the exterior product composition we described previously. We may simply say that a directed linear extension assumes the role of a temporal order for another independent linear extension to be composed with it by means of the exterior product. Equivalently, the replication procedure itself may be thought of as an one-parameter flow over the considered temporal order. Notice also that the origin of this order does not have to be fixed a priori, since the replication flow of an independent directed linear extension along it does not depend on where the origin is positioned, or else, it is of an affine character.

If we take the universality property into account, we realize that the exterior algebra pertaining to a directed linear extension, as above, is isomorphic with the $\mathbb{K}$-algebra of dual numbers over $\mathbb{K}$. More precisely, we have the isomorphisms:

$$
E(\mathbb{K} e) \cong \mathbb{K} \oplus \mathbb{K} \cdot \varepsilon \cong \frac{\mathbb{K}[\varepsilon]}{\left(\varepsilon^{2}\right)}
$$

where the square of $\varepsilon$ is zero, $\varepsilon^{2}=0$. The important fact is that $E(\mathbb{K} e)$ is a commutative algebra over $\mathbb{K}$. Note that the nullity of the exterior product operation of the single directed linear extension $e$ with itself, i.e. $e \wedge e=0$ implies that the directed area of $e$ along itself is zero. Thus, the non-commutative exterior product has a commutative shadow in the exterior algebra $E(\mathbb{K} e)$ expressed by the unit $\varepsilon$ whose square vanishes.

We identify this unit as an infinitesimal unit with respect to which the flow over the considered temporal order takes place. This flow is expressed in commutative algebraic, and thus, spectrally geometric terms by means of $E(\mathbb{K} e) \cong \mathbb{K} \oplus \mathbb{K} \cdot \varepsilon$. In turn, this is equivalent to a first order infinitesimal variation or infinitesimal flow along the $\mathbb{K}$-parameterized variable running along the directed linear extension of $e$, interpreted as a temporal order in this direction.

We conclude that the exterior product operation applied to a single directed linear extension $e$ is reflected in the commutative algebraic shadow of this extension as an ordinary multiplication of its scalar image $\varepsilon$, and moreover, the nullity of the directed area of $e$ along itself is reflected as the vanishing of $\varepsilon^{2}$, granting it the role of an infinitesimal unit with respect to which an one-parameter potential flow along the temporal order of $e$ 's directed extension can be initiated. 
Equivalently phrased, and conclusively, directed linear extensions are reflected as infinitesimals in their respective commutative spectral shadows.

We stress that the non-commutativity of the exterior product operation applied to two independent directed linear extensions is due to the antisymmetry in the order of their composition, which gives rise to a specific orientation (clockwise or anticlockwise) of the completed parallelogram area. The crucial thing in this case is that each of these directed linear extensions serves as a temporal order for the transportation or flow of the other along its extension.

Therefore we have two distinct temporal orders with respect to which the parallelogram area can potentially be completed, but they are not equivalent, they differ in the way they induce an orientation on this area. Let us consider two independent directed linear extensions $e_{1}$ and $e_{2}$, then the ordered composition $e_{1} \wedge e_{2}$ stipulates the replication of $e_{2}$ a total of $\left\|e_{1}\right\|$ times along the linear extension of $e_{1}$, considered as a temporal order for this purpose. In the course of this replication procedure, whereas we may think of a transportation from a determinate point to another determinate point along the linear extension of $e_{1}$ in terms of a sharply defined scalar parameterizing variable, the directed segment $e_{2}$ has to be taken in its totality, in other words, as a potentially simultaneous whole. This means that it assumes potentially all the points of its linear extension in a kind of "tautochrone" superposition. In this sense, it is objectively totally indeterminate in the course of its replication along the determinate extension of $e_{1}$ from point to point. To be sure, the directed linear extension of a segment is not considered to consist of determinate spatial points, rather the latter are implicitly instantiated in the process of replicating another directed segment along the former's extension. Precisely analogous considerations pertain to the oppositely oriented case, referring to the ordered composition $e_{2} \wedge e_{1}$, which requires the replication of $e_{1}$ a total of $\left\|e_{2}\right\|$ times along the linear extension of $e_{2}$, considered as a temporal order in its turn.

The above procedure actually constitutes the cornerstone of the partial derivation method underlying geometric analysis, that is to say, the two-variable geometric calculus expressed in terms of differential forms and their integration, which is generalized to multiple variables by means of the rules of exterior products. In this way, the geometric manifestation of the exterior product in terms of an one-parameter flow along a directed linear extension is expressed locally by way of a partial derivation along this extension, i.e. a one-parameter partial 
differentiation procedure. The fact that the differentiation procedure can be enacted in two ways that differ by a sign is expressed by the Leibniz rule for differentiating products. The Leibniz rule of differentiation is a formal expression of the replication procedure we have already described.

Given that directed linear extensions are reflected as infinitesimals in their respective commutative spectral shadows, we need also to grasp in what way the infinitesimal commutativity underlying the mixed partial derivations with respect to the two independent temporal orders, in the case of two composed linear extensions, reflects the noncommutativity of the exterior product of these extensions, which finally is manifested as a sign difference in the orientation of the composed parallelogram area.

Up to present, we have already seen that the nullity of the exterior product of a single directed linear extension $e$ with itself, $e \wedge e=0$, has a commutative shadow in the exterior algebra $E(\mathbb{K} e)$ expressed by the unit $\varepsilon$ whose square vanishes. We have identified this unit as an infinitesimal unit, with respect to which the flow over the considered temporal order takes place and expressed in commutative algebraic, and thus, spectrally geometric terms, by means of $E(\mathbb{K} e) \cong \mathbb{K} \oplus \mathbb{K} \cdot \varepsilon$. The pertinent question is how the commutative shadow displays in case of two independent linear extensions. An answer to that will allow an easy generalization in all cases involving any finite number of independent linear extensions.

In the context of the above, consider two independent linear extensions in a two-dimensional vector space $V$ over the real numbers. Then, we may define a bilinear map $\rho: V \times V \rightarrow V \otimes V$, where $V \times V \cong V \oplus V$, such that

$$
\left(e_{1}, e_{2}\right) \mapsto \rho\left(e_{1}, e_{2}\right)=e_{1} \otimes e_{2}-e_{2} \otimes e_{1}
$$

Clearly, we obtain that $\rho\left(e_{1}, e_{1}\right)=0, \rho\left(e_{2}, e_{2}\right)=0$, thus $\rho$ is alternating. Therefore, a unique linear map exists in this case $\chi: \wedge^{2} V \rightarrow V \otimes V$, such that:

$$
e_{1} \wedge e_{2} \mapsto \chi\left(e_{1} \wedge e_{2}\right)=e_{1} \otimes e_{2}-e_{2} \otimes e_{1}
$$

Next, we consider the same vector space $V$ and its exterior algebra $E(V)$. The latter is an $\mathbb{R}$-algebra generated by the images of $e_{1}$ and $e_{2}$, denoted by $\hat{\varepsilon}_{1}, \hat{\varepsilon}_{2}$, subject to the relations $\hat{\varepsilon}_{1}^{2}=0, \hat{\varepsilon}_{2}^{2}=0$, and $\hat{\varepsilon}_{1} \hat{\varepsilon}_{2}=-\hat{\varepsilon}_{2} \hat{\varepsilon}_{1}$. Note that $\hat{\varepsilon}_{1}, \hat{\varepsilon}_{2}$ anti-commute, and thus $E(V)$ is a non- 
commutative $\mathbb{R}$-algebra in striking contrast to the case of a single directed linear extension.

Therefore, the exterior algebra $E(V)$ cannot be considered as the infinitesimally-generated commutative shadow we are looking for here. Note that, generally speaking, the commutative shadow always admits of a local description, since infinitesimal quantities are meaningful only within a local context. Globally only their integrated products are observable.

Analogously to the case of a single directed linear extension the sought-after commutative shadow should be thought of as a commutative extension with respect to two infinitesimal units, $\varepsilon_{1}, \varepsilon_{2}$, together with a mixed commutative term of the form $\varepsilon_{1} \cdot \varepsilon_{2}=\varepsilon_{2} \cdot \varepsilon_{1}$ as follows:

$$
\tilde{E}(V) \cong \mathbb{R} \oplus \mathbb{R} \cdot \varepsilon_{1} \oplus \mathbb{R} \cdot \varepsilon_{2} \oplus \mathbb{R} \cdot\left(\varepsilon_{1} \cdot \varepsilon_{2}\right)
$$

where the squares of both $\varepsilon_{1}$ and $\varepsilon_{2}$ are zero, i.e. $\varepsilon_{1}^{2}=0, \varepsilon_{2}{ }^{2}=0$, and the two infinitesimal units commute, i.e. they are scalars in the commutative $\mathbb{R}$-algebra $\tilde{E}(V)$. Note that instead of the field $\mathbb{R}$ considered here, we may similarly think of another field $\mathbb{K}$.

Note that $V$ may written equivalently in the form $V=\mathbb{R} e_{1} \oplus \mathbb{R} e_{2}$, since we are dealing with two independent linear extensions. Then, $E(V)$ as a vector space over $\mathbb{R}$, denoted by $\bar{E}(V)$ to distinguish it from the same space equipped with the non-commutative algebra structure $E(V)$, takes the form:

$$
\bar{E}(V) \cong \bar{E}\left(\mathbb{R} e_{1} \oplus \mathbb{R} e_{2}\right) \cong E\left(\mathbb{R} \varepsilon_{1}\right) \otimes E\left(\mathbb{R} \varepsilon_{2}\right)
$$

We notice that both $E\left(\mathbb{R} \varepsilon_{1}\right)$ and $E\left(\mathbb{R} \varepsilon_{2}\right)$ are not only vector spaces, but they are commutative algebras over $\mathbb{R}$. Thus, $\bar{E}(V)$ is also a commutative $\mathbb{R}$-algebra with respect to the standard $\mathbb{R}$-tensor product, identical with the algebra $\tilde{E}(V)$. Thus, the exterior algebra $E(V)$ has a commutative spectral shadow represented by the algebra $\tilde{E}(V)$.

The significance of the commutative spectral shadow $\tilde{E}(V)$ of $E(V)$ consists in the fact that the unique linear map from the top exterior power in this case, i.e. $\chi: \wedge^{2} V \rightarrow V \otimes V$, such that: 


$$
e_{1} \wedge e_{2} \mapsto \chi\left(e_{1} \wedge e_{2}\right)=e_{1} \otimes e_{2}-e_{2} \otimes e_{1}
$$

descends in the commutative shadow to the map

$$
e_{1} \wedge e_{2} \mapsto \varepsilon_{1} \cdot \varepsilon_{2}-\varepsilon_{2} \cdot \varepsilon_{1}
$$

Therefore, $e_{1} \wedge e_{2}$ belongs to the kernel of this unique linear map after its descent to the commutative shadow $\tilde{E}(V)$. Hence, the commutativity condition of the infinitesimals $\varepsilon_{1}$ and $\varepsilon_{2}$ is obtained, given as $\varepsilon_{1} \cdot \varepsilon_{2}=\varepsilon_{2} \cdot \varepsilon_{1}$, which is nothing else but the standard commutation rule of the mixed partial derivatives.

There is an analogous relation here. Just as the non-commutative exterior algebra $E(V)$ is an algebraic encoding of Grassmann's lineal extension process from layer to layer, starting from directed linear extensions, where the scalars or points are only implicitly assumed, so the commutative spectral shadow $\tilde{E}(V)$ affords a conceptual inversion, in the form of a decoding of this process, which takes place in terms of commuting infinitesimal units, where each one corresponds to an infinitesimal directed extension, culminating in the determination of points according to distinct linear infinitesimal extensions that become dependent at that point.

The crucial aspect of this metaphora is that points are only implicit in the ordered extension process, in the sense that they are instantiated in the procedure of replicating a linear extension along another independent one. They become explicit only in the inversion of the extension in the commutative shadow, and only locally and infinitesimally, by means of making two infinitesimal flows in distinct directions dependent at that point. In this manner, a point becomes explicit as an element bounded by distinct temporal orders manifested as infinitesimal directional flows that meet or become dependent at that point. The necessary condition for the temporal explication of points in this way, according to two different or even a whole multiplicity of temporal determination modes, is that the units of these flows becoming dependent at a point-instant locally commute in the infinitesimal vicinity of that point.

Henceforth, the lineal extension process cannot be conceived independently of its inversion, taking place by means of the commutative spectral shadow of the exterior algebra. In point of fact, in an attempt to reach beyond the exterior multiplication product, called a progressive product, bearing the analogous role of the logical join operation, Grassmann strived for the definition of another type of product, called a 
regressive product, bearing a role analogous to the logical meet operation, and thus constituting the inversion of the former. There is a not a clear consensus in the literature regarding the definition of the regressive product. The proposed metaphora from the non-commutative domain to the commutative spectral shadow of this domain, instantiated as it is infinitesimally, shows that Grassmann's lineal extension cannot be understood independently of Leibniz's infinitesimal analysis. RESOLUTION OF A POINT

It is worth emphasizing the potent, and non-contradictory, indirect selfreferential strategy underlying the conception of points or scalars according to this metaphora. It initiates by means of an ascent from the layer of independent directed linear extensions, where points are only implicitly assumed, to the layer of oriented areas, volumes and so on, being followed by a descent back to lower and lower layers, and culminates in the explication of points as bounds of distinct temporal orders becoming dependent infinitesimally at that point. This type of indirect selfreferentiality arising from the process of ascending and then descending back in order to explicate temporally and multi-linearly what a point is in the context of Grassmann's geometric lineal analysis, is not void. Quite the reverse, it contains the germ of cohomological analysis in general topological spaces or even categorical sites, arguably, one of the most powerful and fruitful methods of modern mathematics and theoretical physics.

There are three basic conceptual issues underlying the dramatic generalization of this metaphora. The first issue pertains to the generalization of Grassmann's exterior algebra from the context of vector spaces defined over a field, to the context of locally free modules defined over an algebra of observables (in particular, locally free sheaves of modules defined over a sheaf of algebras of observables). The second issue pertains to conceptualizing algebraically the previously described metaphora as a process of spectral resolution of the constant scalars. The third issue pertains to viewing this process of resolution of the constants in terms of the notion of an algebraic-topological complex. The definition of the latter is based on the action of an operator, called the coboundary or, exterior derivation operator $\mathbf{d}$, characterized by the property that $\mathbf{d}^{2}=0$, i.e. it is a nilpotent operator of the second degree.

The notion of the coboundary operator that effects differentiation in this setting, is based precisely on the articulation of a point as a bound of distinct temporal orders becoming dependent infinitesimally, i.e. in terms of commuting one-parameter infinitesimal flows, at that point. The specification of the coboundary operator can actually be extracted from 
the constitution of the commutative shadow $\tilde{E}(V)$ of the exterior algebra $E(V)$.

The idea is to unfold the infinitesimal irreducible parts of $\tilde{E}(V)$ as modules, or simply vectors spaces, of differentials of different orders. In particular, in the two-dimensional case we have examined in detail, we consider the vector space of differentials of the first order, which contains only the pure infinitesimal units $\varepsilon_{1}$, and $\varepsilon_{2}$, and then, the vector space of differentials of the second order, which contains the mixed terms. Since the latter are vector spaces of differentials of the first and second order correspondingly their elements, while infinitesimals, are directed geometric magnitudes. Then, we may symbolically consider

$$
\begin{gathered}
\mathbf{d}=\varepsilon_{1} \hat{\varepsilon}_{1}+\varepsilon_{2} \hat{\varepsilon}_{1} \\
\mathbf{d}^{2}=\mathbf{d}(\mathbf{d})=\mathbf{d}\left(\varepsilon_{1} \hat{\varepsilon}_{1}+\varepsilon_{2} \hat{\varepsilon}_{1}\right)=\left(\varepsilon_{1} \cdot \varepsilon_{2}\right) \hat{\varepsilon}_{1} \hat{\varepsilon}_{2}+\left(\varepsilon_{2} \cdot \varepsilon_{1}\right) \hat{\varepsilon}_{2} \hat{\varepsilon}_{1}=\left(\varepsilon_{1} \cdot \varepsilon_{2}-\varepsilon_{2} \cdot \varepsilon_{1}\right) \hat{\varepsilon}_{1} \hat{\varepsilon}_{2}=0
\end{gathered}
$$

which demonstrates the "coboundary law" of constitution of a complex of vector spaces in cohomology theory. Note that the coboundary operator is defined locally, whose purpose is to resolve the points, or else the constant scalars, by way of the concomitant ascent-descent process of Grassmann's lineal geometric analysis.

In the context of locally free modules defined over observable algebras the "coboundary law" expresses the law of "inertial variation" in physical terminology. The discrepancy from inertial variation in the transition from the local to the global conceived topologically, is measured by certain equivalence classes, called cohomology classes. These classes are defined precisely through the notion of coboundary, and they capture in infinitesimal commutative terms some invariant global aspect obtained by their integration.

\section{7 \\ COHOMOLOGY: GROUP SHEAVES AS COEFFICIENT SYSTEMS FOR CALCULUS}

The far-reaching applicability of Grassmann's lineal geometric analysis can be adequately appreciated only if it can be raised appropriately from the level of a vector space to the level of a "locally free module", defined over a smooth manifold or, more generally, over an arbitrary topological space, called accordingly a vector sheaf. The essential aspect of this generalization marking the powerful unifying combination of methods and ideas from the fields of geometry, topology, analysis, and homological algebra, is the notion of localization of a mathematical form, which reaches its greatest heights with the invention of sheaf theory and its subsequent application in the machinery of cohomology theory. 
Conceptually, the invariant transference of Grassmann's lineal extension framework from the level of vectors to the level of locally free modules, necessitates the consideration of locally-definable and variable mathematical entities. At the first stage, the natural generalization of the notion of a vector space defined over a field of scalars is carried over by the notion of a module defined over a commutative algebra of scalars in which the underlying field of definition of this algebra is injected as the constant scalars. The scalars of the algebra may be thought of in a physical sense as the observable quantities.

In this sense, they assume at least the role of continuous functions defined over a topological space. For instance, the algebra of scalars may be generally considered as the $\mathbb{R}$-algebra, or more generally $\mathbb{C}$-algebra, of continuous functions evaluated in this field. The notion of evaluation also bears a physical semantics, since it refers to the evaluation of the observables at the states of the base topological space, that is, the recording of events in terms of values in the field of definition. Since the vectorial quantities defined over the algebra of scalars should be in principle expressible in terms of a basis, as in the case of vector spaces, the modules are required to be finitely generated or free.

Notwithstanding this fact, there are two essential issues that have to be dealt with in this generalization:

The first pertains to the realization that the operative manifestation of Grassmann's lineal geometric analysis requires both the commutativity, and the infinitesimally-generated nature of the algebraic structural shadow of the extension process. In the context of the generalization we are dealing with the base field is substituted by the algebra of scalars, whereas the infinitesimals of all orders are included as ideals in this commutative shadow. In an analogous fashion, they give rise to modules of differentials, that is, continuous differential forms of various orders, such that the pertinent coboundary operator is defined in terms of them. In this way, the latter may be simply identified as an exterior derivative operator from the algebra of scalars to the modules of differentials of the first degree obeying the Leibniz rule, and subsequently generalized to all higher order by means of Grassmannian calculus, such that the coboundary law is satisfied. Technically, the base topological space is assumed to be paracompact and Hausdorff.

The conceptual problem arising in this setting is that continuous functions are defined by their values at the points of the base topological space. This is quite at odds with the unfolding of Grassmann's geometric analysis, since the points are only implicitly assumed in the initiation of the extension process, whereas they are actually explicated as bounds of criss-crossing temporal orders becoming dependent at a point, and most importantly, this is enacted only in terms of infinitesimal flows. The latter are only locally defined, they just require a local cover of this point. 
Therefore, to introduce here, in the generalized scheme of geometric analysis scalars as continuous functions determined by their values at points of the assumed topological space would be to beg the question. There is a way around this serious issue that makes the generalization of Grassmanian analysis proceed in a meaningful way preserving the unfolding semantics we have explicated previously.

The essentially simple idea required for this purpose, notably also of a physical descent, is to consider the localization of the whole scheme. This is something already mandated from the origin of Grassmannian analysis, since the commutative shadow of the non-commutative exterior algebra, generated as it is infinitesimally can only be of a local character. This localization procedure of all scalar and vectorial quantities as well as geometric forms bears the name of sheaf theory.

Instead of thinking in terms of globally defined continuous functions or forms of any order, we think in terms of locally defined functions, called sheaf sections, and their corresponding germs, as detailed in the preceding. Accordingly, the base topological space is considered only implicitly in the beginning of geometric analysis, with no reference required at all on its point determination. Instead what matters is the local covering structure of this space, which consists of the open sets covering the implicitly assumed points, that become explicable only in the culmination of the analysis in terms of the commutative shadow, or equivalently the algebra of scalars together with the cohomological temporal unfolding of the coboundary operator to higher orders of infinitesimal germs to be integrated appropriately as potential bearers of global information. Thus, under this essential localization requirement, the generalization of geometric analysis takes place in terms of a sheaf of commutative algebras of scalars or observables and locally free sheaves of modules defined over it so that the notion of basis persists in this context as well.

The second issue pertaining to this generalization has to do with the doubly articulated semantics of the unfolding of geometric analysis given the necessary condition of topological or sheaf-theoretic localization, as established. Since, the point structure of the base topological space is only implicitly assumed what really matters is what plays the role of a topology, i.e. the role of a local covering structure of the points that allows the formulation of the concept of a continuous function with respect to these covers. Traditionally, this is implemented in terms of a collection of open covers of a topological space that is closed under arbitrary unions and finite intersections.

Grothendieck generalized the notion of a covering family consisting of open sets by the notion of a covering sieve, not necessarily consisting of injective open covers, but allowing the definition of continuity in these generalized terms. The idea of generalized covers is 
inspired essentially by Riemann's covering principle together with its reconceptualization from the perspective of Galois theory.

In a Grothendieck topology the open covers of a space are maps into this space, where instead of their pairwise intersections we have to look at their fibered products, or pullbacks, while unions play no essential role. In this conception, the idea of a cover is thought of as an observation horizon of a point, represented as an opening in a sieve that infiltrates observable information compatibly at different resolution layers by refinement until its temporal completion, thanks to which the essence of a point as a bearer of some globally irreducible, quantifiable, and invariant information may be unfolded cohomologically. The defining requirements of these generalized covers are the following: Covers are transitive meaning that covers of covers are also covers; covers are stable under pullback operations conforming to the stability of the notion of a cover under change of base; and finally, isomorphisms are qualified as covers.

Note that the present type of cohomological unfolding is not typically the same as the one implemented by Grassmanian analysis in terms of differential forms, or more precisely, de Rham cohomology. It is a differently conceived kind of unfolding by means of another suitably defined coboundary operator, called Čech cohomology. Recalling that the notion of a coboundary operator is the encoding of a multiplicity of pointbounding temporal orders in infinitesimal terms, subject to the coboundary law, the conception of Čech cohomology is based on a different articulation of what a temporal order is, independently of the concept of a local linearly extended geometric magnitude or form as in the de Rham case.

The amazing fact that these two different types of cohomological unfolding can be unified, an insight that lies deeply at the heart of local gauge invariance that will be expanded later, is based on Grothendieck's vision that a locally variable sheaf of coefficients is actually the natural argument of all cohomological theories in this context.

In other words, the natural argument of a cohomology theory is not just a space, as it was initially thought of, but a space together with an observable sheaf of coefficients, such that the space constitutes the observed spectrum of the sheaf employed cohomologically for this purpose. The global nature of points of this spectrum is typically determined, as it is actually expected, by a constant sheaf of coefficients, namely, a sheaf of locally constant sections valued in the integers, or the reals, or the complex numbers. In this sense, a commutative observable sheaf of coefficients plays locally the equivalent role of a measurement procedure, or apparatus in physical terminology, that is capable of capturing some global invariant feature only by cohomological means.

In Grothendieck's phraseology, we read the following excerpt (in English translation) from his autobiographical reflections contained in Récoltes et semailles: Réflexions et témoignages sur un passé de 
mathématicien: "Consider the set formed by all sheaves over a (given) topological space or, if you like, the formidable arsenal of all the "rulers" that can be used in taking measurements on it. We will treat this "ensemble", or "arsenal" as one equipped with a structure that may be considered "self-evident", one that crops up "in front of one's nose": that is to say, a Categorical structure ... It functions as a kind of "superstructure of measurement", called the "Category of Sheaves" (over the given space), which henceforth shall be taken to incorporate all that is most essential about that space. This is in all respects a lawful procedure, (in terms of "mathematical common sense") because it turns out that one can "reconstitute" in all respects, the topological space by means of the associated "category of sheaves" (or "arsenal" of measuring instruments").

Having already described the process of continuous unfolding from the local to the global achieved by the theory of sheaves, it is worthwhile for our purposes to focus on the basic ideas of Čech cohomology theory, as a basic predecessor of the later developments. This is particularly interesting in relation to the notion of double articulation laid out briefly above, since it introduces a novel way of thinking about the global nature of the points of a topological space in terms of the idea of coverings, which for reasons of simplicity, we may currently identify with the typical open covers of a topological space, whose global aspects are only implicitly assumed $a b$ initio.

The basic characteristic of these open coverings is that they are partially ordered by inclusion. Note that this is just a partial and not total order of open covers, meaning that it is capable of subsuming a variety of potential local directed total orders or chains. The crucial idea is to force an inductive system out of these open covers that is capable of resolving points in terms of a corresponding dual projective system of Abelian groups of locally defined function elements, more precisely sections of a sheaf, over these covers at varying resolution horizons. Note that a section is conceived extensively as a whole over its domain or locus of definition.

In the initial conception of this theory these function elements were considered constants, for instance constant real-valued functions, or the constant coefficient system $\mathbb{R}$. In this sense, an extension of function elements is initiated by gluing them together through a process of joining together open covers, which has to be compatible with the inverse process of restriction of these elements to smaller open covers. For this purpose, it is required that for any pair of them there exists an infimum expressed by their intersection, or meet, or more generally pullback operation, such that their overlap is totally contractible. Clearly, for every sub-collection of these covers there also exists a supremum with respect to their partial order relation. This implicitly also leads us to posit 
a minimal cover to serve as the inductive limit of all pairwise intersections of all covers forming a chain.

Thinking in temporal terms, we seek an articulation of points through this extension-restriction process in terms of an infinitesimally, and thus locally, generated commutative shadow, which can be equivalently expressed by means of a locally variable sheaf of coefficients (which can obviously be the constants) together with an appropriately defined coboundary operator. The latter should act on cochains of function elements in such a way that all function elements in the image of its action should form coboundaries, that is, local infinitesimallygenerated flows over the directed temporal orders represented by the respective dual chains of covers, called in turn boundaries.

The first thing to note is that although coboundaries are locally defined entities, boundaries are globally defined entities with respect to the implicit base topological space. Thus although the coboundary operator is a locally defined operator on cochains, its dual boundary operator is a globally defined operator on chains. This eventually leads to the marking difference between cohomological and homological entities providing the appropriate hint required to pair them together in a suitable manner. At this stage, there is a second important thing we should also take into account, namely that in order that coboundary infinitesimal flows over distinct directed temporal orders can bound points by means of crisscrossing, they should be clearly alternating. Thus, both chains and cochains should be directed in the order-theoretic sense and alternating; something that it is implicitly assumed from the beginning usually, but has to be stressed at this stage.

The most important idea now is that in a topological space, in sharp distinction to a linear space, this procedure does not exhaust the ontology of points. The reason is that this implicitly postulated global topological space is generally neither contractible, nor simply connected. Thus, there appears a new kind of ontology of points, which may be characterized in terms of a variety of singular points, for instance holes, singularities, poles, branching points, sources, residues, degeneracies, foams, and so on. All these types of singular points should be accounted for indirectly, through metaphora, in terms of some global invariant information they eventually give rise to with respect to the local commutative spectral shadow, instantiated by the locally variable sheaf of coefficients together with the locally defined coboundary operator. Since the global nature of these singular points cannot be probed and exhausted at the infinitesimal level, meaning that singular points cannot be bounded via crisscrossing infinitesimal flows locally, the type of cochains needed for their description cannot be coboundaries as in the former case, but cochains which are annihilated by the coboundary operator, or equivalently cochains that are vanishing over boundary chains, called in turn cocycles. 
In this case, the crucial issue is that we require a novel notion of directed temporal order to be applied for distinguishing singular points, of a different quality from the one conceptualized in terms of boundary chains. It is intuitive enough to think that a singular point, since it cannot be bounded by infinitesimal flows meeting at this point, can only be amenable to a process of repeated circulations around it, where all these circulations are not equivalent, but are in principle distinguishable in terms of different global attributes.

Dually thinking, the type of global chains needed for this purpose, called cycles, are clearly not boundaries, and most significantly, they encapsulate a novel type of temporal order that it is qualitatively different from the former one. More precisely, the type of temporal order encapsulated by cycles is characterized in terms of the periods of the locally-generated process of cocyclic circumscription, to be thought of as a global integration procedure, and not in terms of instants as in the former case.

The only issue remaining after making this qualitative difference is to discern the internal relation that cocycles bear with coboundaries, or equivalently cycles with boundaries. We can immediately see that due to the validity of the coboundary law for alternating cochains, all coboundaries are trivially cocycles as well. Equivalently, all boundary chains are trivially cycles as well, showing that the spectral unveiling of singular points is deeper than that of standard points, since the type of temporal order needed to account for the latter is trivially subsumed by the former.

Hence, at each degree, we may consider the quotient of the space of all cochains that are cocycles by the space of all cochains that are coboundaries to obtain the notion of a corresponding cohomology class. From this viewpoint, we realize that coboundaries function as bridges in statu-nascendi for the transition from one cocycle to another of the same cohomology class. We conclude that it is the concept of a cocycle that epitomizes the notion of a gauge in a topological context, whence the notion of gauge invariance is expressed through the concept of a cohomology class, as we shall elaborate in more detail later.

We proceed with a brief technical exposition to accompany the above conceptual undertaking of Eduard Čech theory. We emphasize that this cohomology theory is based on the intersection properties of open covers. Let $\mathbb{U}=U_{k}, k \in K$, be a system of open covers of the implicitly assumed global topological space $X$. For $k_{1}, k_{2}, \ldots, k_{n} \in K$, we denote:

$$
U_{\kappa}:=U_{k_{0} \ldots k_{n}}:=U_{k_{0}} \cap \ldots \cap U_{k_{n}}
$$


and define a degree $n$-cochain with real coefficients for the covering as a totally ordered collection of function elements $\phi:=\left\{f_{\kappa}\right\}$, where $\kappa$ is totally ordered with $n+1$ elements, and each $f_{\kappa}$ is constant, realvalued and alternating.

We denote the set of all degree $n$-cochains with real coefficients obtained from a covering $\mathbb{U}$ of $X$ by $C^{n}(X ; \mathbb{R} ; \mathbb{U})$. Then, pointwise addition over the real numbers makes $C^{n}(X ; \mathbb{R} ; \mathbb{U})$ an Abelian group, and scalar multiplication provides it with the structure of a real vector space. According to the above definition, an element of $C^{0}(X ; \mathbb{R}, \mathbb{U})$ corresponds to the assignment of a constant real-valued function to each open cover in $\mathbb{U}$. We consider that the covering $\mathbb{U}$ is finite, say of cardinality $v$, hence we obtain $C^{0}(X ; \mathbb{R}, \mathbb{U})=\mathbb{R}^{v}$. Analogously, the elements in $C^{1}(X ; \mathbb{R}, \mathbb{U})$ correspond to constant real-valued functions defined on the overlap of two open covers, and so on.

The coboundary map, or Čech differential is a linear map

$$
\delta: C^{n}(X ; \mathbb{R}, \mathbb{U}) \rightarrow C^{n+1}(X ; \mathbb{R}, \mathbb{U})
$$

for every index $n>0$, which sends each $n$-cochain $\phi$ to a $n+1$ cochain $\delta \phi$, i.e. to a coboundary, that is a set of constant real-valued functions defined on intersections of $n+2$ open covers, each expressed as an alternating sum of restrictions of $\phi$ 's to these covers. The coboundary operator by construction satisfies the coboundary law, $\delta^{2}=0$.

We say that a cochain $\phi$ in $C^{n}(X ; \mathbb{R}, \mathbb{U})$ is closed, or a cocycle, if $\delta \phi=0$, whereas we say that a cochain $\varphi$ in $C^{n}(X ; \mathbb{R}, \mathbb{U})$ is a coboundary if it is in the image of the coboundary operator $\delta$, i.e. there exists a cochain $\chi$ in $C^{n-1}(X ; \mathbb{R}, \mathbb{U})$, such that $\delta \chi=\varphi$. Then, we define the $n$-cohomology Abelian group of equivalence classes of cocycles modulo coboundaries with coefficients in $\mathbb{R}$, as the quotient:

$$
H^{n}=\frac{\operatorname{Ker}\left(\delta: C^{n} \rightarrow C^{n+1}\right)}{\operatorname{Im}\left(\delta: C^{n-1} \rightarrow C^{n}\right)}
$$

Superficially, it seems that the above cohomology groups depend on the covering $\mathbb{U}$ chosen. This is not true, but this wrong impression can be 
rectified by applying the inductive limit construction over a refinement of $\mathbb{U}$. Practically, it suffices to consider all open covers of $\mathbb{U}$ which are contractible, so that all their intersections are contractible as well.

It is important to notice that the structure of the real numbers is not constraining in any way for the establishment of Čech's theory. All the arguments can be carried out for constant functions in any Abelian group. It follows that, we obtain Abelian cohomology groups with values in $G$ for any Abelian group of coefficients $G$. Most appropriately, because of the pertinent localization philosophy, we should focus on a locally variable Abelian group structure of coefficients, i.e. an Abelian group sheaf of coefficients, which is usually an Abelian group sheaf of locally constant functions.

After this clarification, we may examine in more detail the structure of the 0 -th Abelian cohomology group $H^{0}(X ; \mathbb{R}, \mathbb{U})$. This group emanates purely from 0 -cocycles, that is, from cochains $\phi:=\left\{f_{\kappa}\right\}$, where $\kappa$ contains only 1 element, and each $f_{\kappa}$ is a locally constant, real-valued function. We have:

$$
\delta \phi=0 \Leftrightarrow f_{a}=f_{b}
$$

on the intersection $U_{a} \cap U_{b}$ of the open covers $U_{a}$ and $U_{b}$.

Therefore, $H^{0}(X ; \mathbb{R}, \mathbb{U})$ is the Abelian group of locally constant realvalued functions. In this way, the group $H^{0}(X ; \mathbb{R}, \mathbb{U})$ detects the connected components of the topological space $X$. If $X$ is connected then $H^{0}(X ; \mathbb{R}, \mathbb{U})=\mathbb{R}$. In this case, $H^{0}(X ; \mathbb{R}, \mathbb{U})$ is identified as the Abelian group of all globally defined constant functions.

Let us now examine the 1-st Abelian cohomology group $H^{1}(X ; \mathbb{R}, \mathbb{U})$. This group emanates from 1 -cocycles, i.e. from cochains $\phi:=\left\{f_{\kappa}\right\}$, where $\kappa$ contains 2 elements, and each $f_{\kappa}$ is a locally constant, real-valued function. Equivalently, we consider the family $\phi:=\left\{f_{a b}: U_{a} \cap U_{b} \rightarrow \mathbb{R}\right\}$, such that $f_{a b}=-f_{b a}$, and the following cocycle relation is satisfied:

$$
\delta \phi=0 \Leftrightarrow f_{a c}=f_{a b}+f_{b c}
$$


on the triple intersection of open covers $U_{a} \cap U_{b} \cap U_{c}$. These 1-cocycles $\phi$ yield cohomology classes in $H^{1}(X ; \mathbb{R}, \mathbb{U})$ by taking them up to the addition of a coboundary. Equivalently, for any two cocycles $\phi, \phi^{\prime}$, we have that:

$$
[\phi]=\left[\phi^{\prime}\right] \Leftrightarrow f_{a b}^{\prime}=f_{a b}+f_{a}-f_{b}
$$

for some 0 -cochain $\left\{f_{a}: U_{a} \rightarrow \mathbb{R}\right\}$.

After our interlude among the workings of Čech cohomology theory, the essential thing to keep in mind is that the natural argument of a cohomology theory is not a global topological space or a manifold, which after all is initially only implicitly posited, but a space together with an observable sheaf of coefficients, such that the point constitution (both standard and singular) of the former arises as the spectrum of the sheaf cohomological analysis. It is an established fact that in the case of paracompact topological spaces the calculation of cohomology with coefficients into a typical sheaf of coefficients is equivalent to the calculation of Čech cohomology theory with values in the corresponding sheaf (complete presheaf).

In this context, the calculation of cohomology with values in a constant group sheaf is of particular significance; such a group sheaf consists of locally constant sections, as well as the interrelation among constant sheaves in their function as cohomology coefficients. The most important tool in this respect, is provided by the exponential short exact sequence of constant group sheaves. The validity of this exact sequence of group sheaves conceptually descents from Riemann's covering principle, that we have already seen in the context of complex function theory on Riemann surfaces in relation to the complex exponential function and its local inverse complex logarithm function.

In terms of constant Abelian group sheaves, we have locally a certain interrelation of coefficients, expressed via the exact sequence:

$$
\begin{array}{r}
0 \rightarrow \mathbb{Z} \stackrel{\iota}{\longrightarrow} \mathbb{C} \stackrel{\exp }{\longrightarrow} \tilde{\mathbb{C}} \rightarrow 1, \\
\operatorname{Ker}(\exp )=\operatorname{Im}(\imath) \cong \mathbb{Z}, \quad(2)
\end{array}
$$

where $\mathbb{Z}$ is the constant additive Abelian group sheaf of the integers, $\mathbb{C}$ is the constant additive Abelian group sheaf of the complexes, and $\tilde{\mathbb{C}}$ is the constant multiplicative Abelian group sheaf of non-zero complexes.

The above exponential short exact sequence can be specialized further to the following short exact sequence of constant Abelian group sheaves: 


$$
0 \rightarrow \mathbb{Z} \stackrel{\imath}{\longrightarrow} \mathbb{R} \stackrel{\exp (2 \pi i)}{\longrightarrow} \mathbb{U}(1) \rightarrow 1
$$

where $\mathbb{R}$ is the constant additive Abelian group sheaf of the reals, and $\mathbb{U}(1)$ is the constant multiplicative Abelian group sheaf of unit modulus complexes (phases).

The applicability of Grassmann's lineal geometric analysis is finally generalized and empowered to its full strength under the program of sheaf-theoretic localization, that marks the essential semantic transition from the level of vector spaces to the level of vector sheaves. The important thing is that Grassmann's analysis permeates the level of vector sheaves, meaning that it is invariant under localization, or equivalently exhibits a functorial behavior that justifies its universal relevance and applicability. For reasons of completeness, it is worth including the basic definition of the notion of a vector sheaf that characterizes the correlative generalization.

We consider a pair $(X, \mathcal{A})$ consisting of a paracompact (Hausdorff) topological space $X$ and a soft sheaf of commutative rings $\mathcal{A}$ localized over $X$. The above pair is considered as the Gelfand spectrum of a corresponding algebra of observables $A$. We remind that if $\mathbb{C}$ is the field of complex numbers, then an $\mathbb{C}$-algebra $A$ is a ring $A$ together with a morphism of rings $\mathbb{C} \rightarrow A$ (making $A$ into a vector space over $\mathbb{C}$ ) such that, the morphism $A \rightarrow \mathbb{C}$ is a linear morphism of vector spaces. Notice that the same holds if we substitute the field $\mathbb{C}$ with any other field, for instance, the field of real numbers $\mathbb{R}$. We also assume that the stalk $\mathcal{A}_{x}$ of germs is a local commutative $\mathbb{C}$-algebra for any point $x \in X$. A typical example is the case, where $X$ is a smooth manifold of and $\mathcal{A}$ is the $\mathbb{C}$-algebra sheaf of germs of smooth functions localized over $X$. Together with a $\mathbb{C}$-algebra sheaf $\mathcal{A}$ we also consider the Abelian group sheaf of invertible elements of $\mathcal{A}$, denoted by $\mathcal{A}^{\bullet}:=\tilde{\mathcal{A}}$.

An immediate generalization of the exponential short exact sequence of constant Abelian group sheaves is provided by the following short exact sequence of variable Abelian group sheaves, which models sheaf-theoretically the process of exponentiation in terms of the variable sheaves of coefficients $\mathcal{A}$ and $\tilde{\mathcal{A}}$ :

$$
0 \rightarrow \mathbb{Z} \stackrel{\imath}{\longrightarrow} \mathcal{A} \stackrel{e}{\longrightarrow} \tilde{\mathcal{A}} \rightarrow 1
$$

where $\mathbb{Z}$ is the constant abelian group sheaf of integers (sheaf of locally constant sections valued in the group of integers), such that: 


$$
\operatorname{Ker}(e)=\operatorname{Im}(\imath) \cong \mathbb{Z}
$$

An $\mathcal{A}$-module $\mathcal{E}$ is called a locally free $\mathcal{A}$-module of finite rank $\mathrm{m}$, or simply a vector sheaf of states, if for any point $x \in X$ there exists an open set $U$ of $X$ such that:

$$
\left.\mathcal{E}\right|_{U} \cong \bigoplus^{m}\left(\left.\mathcal{A}\right|_{U}\right):=\left(\left.\mathcal{A}\right|_{U}\right)^{m}
$$

where $\left(\left.\mathcal{A}\right|_{U}\right)^{m}$ denotes the $m$-terms direct sum of the sheaf of $\mathbb{C}$ observable algebras $\mathcal{A}$ restricted to $U$, for some $m \in \mathbb{N}$. We call $\left(\left.\mathcal{A}\right|_{U}\right)^{m}$ the local sectional frame of $\mathcal{E}$ associated via the open covering $\mathcal{U}=\{U\}$ of $X$.

In case that the rank is 1 , the corresponding vector sheaf is called a line sheaf of states, that is locally for any point $x \in X$ there exists an open set $U$ of $X$ such that:

$$
\left.\left.\mathcal{E}\right|_{U} \cong \mathcal{A}\right|_{U}
$$

Furthermore, if for any point $x \in X$ there exists an open set $U$ of $X$ such that:

$$
\left.\mathbb{S}\right|_{U} \cong \bigoplus^{m}\left(\left.\mathbb{C}\right|_{U}\right):=\left(\left.\mathbb{C}\right|_{U}\right)^{m}
$$

then we call any locally free $\mathbb{C}$-module $\mathbb{S}$ of finite rank $m$, for some $m \in \mathbb{N}$, a complex linear local system of rank $m$.

The notion of a vector sheaf of states generalizes the notion of a vector space in the sense that locally every section of a finite rank vector sheaf can be written as a finite linear combination, or superposition of a basis of sections with variable coefficients from the local observable algebra. For example, if $X$ is a smooth manifold and $\mathcal{A}$ is the $\mathbb{C}$ algebra or $\mathbb{R}$-algebra sheaf of germs of smooth functions on $X$, then every section can be locally written as a finite superposition of a basis of sections with coefficient being real-valued smooth functions. We note that the set of sections of every vector bundle on a topological space (not necessarily a smooth manifold) forms a vector sheaf of sections localized over this space.

Given a vector sheaf of states $\mathcal{E}$, there is specified a Čech 1cocycle with respect to a covering $\mathcal{U}=\{U\}$ of $X$, called a coordinate 1 - 
cocycle in $Z^{1}(\mathcal{U}, G L(m, \mathcal{A})$ (with values in the sheaf of germs of sections into the complex general linear group $G L(m, \mathbb{C})$ ), as follows:

$$
\begin{gathered}
\eta_{\alpha}:\left.\mathcal{E}\right|_{U_{\alpha}} \cong\left(\left.\mathcal{A}\right|_{U_{\alpha}}\right)^{m}, \\
\eta_{\beta}:\left.\mathcal{E}\right|_{U_{\beta}} \cong\left(\left.\mathcal{A}\right|_{U_{\beta}}\right)^{m} .
\end{gathered}
$$

Thus, for every $x \in U_{\alpha}$ we have a stalk isomorphism:

$$
\eta_{\alpha}(x): \mathcal{E}_{x} \cong \mathcal{A}_{x}^{m}
$$

and similarly for every $x \in U_{\beta}$. If we consider that $x \in U_{\alpha} \bigcap U_{\beta}$, then we obtain the isomorphism:

$$
g_{\alpha \beta}(x)=\eta_{\alpha}(x)^{-1} \circ \eta_{\beta}(x): \mathcal{A}_{x}^{m} \cong \mathcal{A}_{x}^{m} .
$$

The $g_{\alpha \beta}(x)$ is thought of as an invertible matrix of germs at $x$. Consequently, $g_{\alpha \beta}$ is an invertible matrix section in the sheaf of germs $G L(m, \mathcal{A})$ (taking values in the general linear group $G L(m, \mathbb{C})$ ). Moreover, $g_{\alpha \beta}$ satisfies the cocycle conditions $g_{\alpha \beta} \circ g_{\beta \gamma}=g_{\alpha \gamma}$ on triple intersections whenever they are defined.

It is clear in this way that we obtain a vector bundle with typical fiber $\mathbb{C}^{m}$, structure group $G L(m, \mathbb{C})$, whose sections form the vector sheaf of states we started with. In particular, for $m=1$, we obtain a line bundle $L$ with fiber $\mathbb{C}$, structure group $G L(1, \mathbb{C}) \cong \tilde{\mathbb{C}}$ (the non-zero complex numbers), whose sections form a line sheaf of states $\mathcal{L}$. Clearly, by imposing a unitarity condition the structure group is reduced to $U(1)$. Thus, particularly in the case of a line sheaf of states we have a bijective correspondence:

$$
\mathcal{L} \leftrightarrow\left(g_{\alpha \beta}\right) \in Z^{1}(\mathcal{U}, \tilde{\mathcal{A}})
$$

where $G L(1, \mathcal{A}) \cong \mathcal{A}^{\bullet}:=\tilde{\mathcal{A}}$ is the group sheaf of invertible elements of $\mathcal{A}$ (taking values in $\tilde{\mathbb{C}}$ ), and $Z^{1}(\mathcal{U}, \tilde{\mathcal{A}})$ is the set of coordinate 1 cocycles. In physical terminology, a coordinate 1-cocycle effects a local frame transformation, or equivalently a local gauge transformation of a vector sheaf of states $\mathcal{E}$. 
Every 1 -cocycle can be conjugated with a 0 -cochain $\left(t_{\alpha}\right)$ in the set $C^{0}(\mathcal{U}, \tilde{\mathcal{A}})$ to obtain another equivalent 1-cocycle:

$$
g_{\alpha \beta}^{\prime}=t_{\alpha} \cdot g_{\alpha \beta} \cdot t_{\beta}^{-1}
$$

If we consider the coboundary operator:

$$
\Delta^{0}: C^{0}(\mathcal{U}, \tilde{\mathcal{A}}) \rightarrow C^{1}(\mathcal{U}, \tilde{\mathcal{A}})
$$

then the image of $\Delta^{0}$ gives the set of 1-coboundaries of the form $\Delta^{0}\left(t_{\alpha}^{-1}\right)$ in $B^{1}(\mathcal{U}, \tilde{\mathcal{A}})$ :

$$
\Delta^{0}\left(t_{\alpha}^{-1}\right):=t_{\alpha} \cdot t_{\beta}^{-1}
$$

Thus, we obtain that the 1-cocycle $g_{\alpha \beta}^{\prime}$ is equivalent to the 1-cocycle $g_{\alpha \beta}$ in $Z^{1}(\mathcal{U}, \tilde{\mathcal{A}})$ if and only if there exists a 0 -cochain $\left(t_{\alpha}\right)$ in the set $C^{0}(\mathcal{U}, \tilde{\mathcal{A}})$, such that:

$$
g_{\alpha \beta}^{\prime} \cdot g_{\alpha \beta}^{-1}=\Delta^{0}\left(t_{\alpha}^{-1}\right)
$$

where, the multiplication above is meaningful in the Abelian group of 1cocycles $Z^{1}(\mathcal{U}, \tilde{\mathcal{A}})$.

Due to the bijective correspondence of line sheaves with coordinate 1 -cocycles with respect to an open covering $\mathcal{U}$, we immediately obtain the following:

The set of isomorphism classes of line sheaves of states over the same topological space $X$, denoted by $I s o(\mathcal{L})(X)$, is in bijective correspondence with the set of cohomology classes $H^{1}(X, \tilde{\mathcal{A}})$ :

$$
\operatorname{Iso}(\mathcal{L})(X) \cong H^{1}(X, \tilde{\mathcal{A}})
$$

Furthermore, each equivalence class $[\mathcal{L}] \equiv \mathcal{L}$ in $I \operatorname{so}(\mathcal{L})(X)$ has an inverse, defined by:

$$
\mathcal{L}^{-1}:=\operatorname{Hom}_{\mathcal{A}}(\mathcal{L}, \mathcal{A})
$$


where, $\operatorname{Hom}_{\mathcal{A}}(\mathcal{L}, \mathcal{A}):=\mathcal{L}^{*}$ denotes the dual line sheaf of $\mathcal{L}$. This is actually deduced from the fact that we can define the tensor product of two equivalence classes of line sheaves over $\mathcal{A}$ so that:

$$
\mathcal{L} \otimes_{\mathcal{A}} \mathcal{L}^{*} \cong \operatorname{Hom}_{\mathcal{A}}(\mathcal{L}, \mathcal{L}) \equiv \mathcal{E} n d_{\mathcal{A}} \mathcal{L} \cong \mathcal{A}
$$

Hence, we conclude that the set of isomorphism classes of line sheaves of states over the same topological space $X, I s o(\mathcal{L})(X)$, has an Abelian group structure with respect to the tensor product over the observable algebra sheaf $\mathcal{A}$, and isomorphically the set of cohomology classes $H^{1}(X, \tilde{\mathcal{A}})$ is also an Abelian group, where the tensor product of two line sheaves of states corresponds to the product of their respective coordinate 1-cocycles. AND INVARIANTS

In the context of Grassmann's lineal geometric analysis we reached the following conclusion: In the same way that the non-commutative exterior algebra $E(V)$ is an algebraic representation of Grassmann's lineal extension process from layer to layer starting from directed linear extensions, where the scalars or points are only implicitly assumed, the commutative shadow $\tilde{E}(V)$ provides an inversion of this process, in terms of commuting infinitesimal units, where each one of them corresponds to a directed extension, culminating in the determination of points according to distinct linear extensions becoming dependent at that point.

More precisely, the constitution of the commutative shadow $\tilde{E}(V)$ of the exterior algebra $E(V)$ is elucidated by means of the locally defined coboundary operator, based on the idea of semantically unfolding the infinitesimal irreducible parts of $\tilde{E}(V)$ as modules or simply vectors spaces of differentials of different orders. In this manner, the notion of the coboundary operator is actually based precisely on the articulation of a point as a bound of distinct temporal orders becoming dependent infinitesimally, that is, in terms of commuting one-parameter infinitesimal flows, at that point.

The coboundary operator gives rise to an exterior derivation operator $d$, characterized by the property that $d^{2}=0$, in other words, it is a nilpotent operator of the second degree. More concretely, the 
exterior derivation operator is thought of as acting on modules of differentials, called differential forms.

In order that Kähler's algebraic extension method becomes suitable as a generator of a universal mechanism of differential geometric analysis it should be susceptible to the process of sheaf-theoretic localization of an observable algebra. In turn, this would allow the complete disassociation of the differential mechanism from any underlying spatial substratum opening up the way for a functorial formulation of differential geometry.

The localization of Kähler's algebraic extension method in sheaf theoretic terms requires first of all the notion of a universal derivation of the observable algebra sheaf $\mathcal{A}$ considered as an algebra sheaf over the constant sheaf of the reals $\mathbb{R}$, or more generally, as an algebra sheaf over the constant sheaf of the complexes $\mathbb{C}$.

The universal $\mathbb{R}$-derivation of the observable algebra sheaf $\mathcal{A}$ to the universal $\mathcal{A}$-module sheaf $\Omega^{1}(\mathcal{A}):=\Omega^{1}$, called the $\mathcal{A}$-module sheaf of differential 1 -forms, is the universal $\mathbb{R}$-linear sheaf morphism (natural transformation) $\partial:=d^{0}$ :

$$
d^{0}: \mathcal{A} \rightarrow \Omega^{1}
$$

such that the Leibniz condition is satisfied:

$$
d^{0}(s \cdot t)=s \cdot d^{0}(t)+t \cdot d^{0}(s),
$$

for any continuous local sections $s, t$ belonging to $\mathcal{A}(U)$, with $U$ an open set in $X$. Notice that all the above definitions are strictly local, which means they are considered stalk-wise. We may also use the following notational convention: $\mathcal{A}:=\Omega^{0}$.

The major ingredient in setting up a universal mechanism of differential geometric analysis is the validity of the Poincaré Lemma:

$$
\operatorname{Ker}\left(d^{0}\right)=\mathbb{R} \text {. }
$$

For each $n \in N, n \geq 2$, the $n$-fold exterior product is defined as follows: $\Omega^{n}(\mathcal{A})=\wedge^{n} \Omega^{1}(\mathcal{A})$ where $\Omega(\mathcal{A}):=\Omega^{1}(\mathcal{A})$. We notice that there exists an $\mathbb{R}$-linear sheaf morphism:

$$
d^{n}: \Omega^{n}(\mathcal{A}) \rightarrow \Omega^{n+1}(\mathcal{A})
$$


for all $n \geq 0$. Let $\omega \in \Omega^{n}(\mathcal{A})$, then $\omega$ has the form:

$$
\omega=\sum f_{i}\left(d l_{i 1} \wedge \ldots \wedge d l_{i n}\right)
$$

with $f_{i}, l_{i j}, \in \mathcal{A}$ for all integers $i, j$. Further, we define:

$$
d^{n}(\omega)=\sum d f_{i} \wedge d l_{i 1} \wedge \ldots \wedge d l_{i n}
$$

From the above, we immediately obtain that the composition of two consecutive $\mathbb{R}$-linear sheaf morphisms vanishes, that is $d^{n+1} \circ d^{n}=0$, abbreviated in the symbolic form $d^{2}=0$, expressing the coboundary law in this context.

The sequence of $\mathbb{R}$-linear sheaf morphisms:

$$
\mathcal{A} \rightarrow \Omega^{1}(\mathcal{A}) \rightarrow \ldots \rightarrow \Omega^{n}(\mathcal{A}) \rightarrow \ldots
$$

is a complex of $\mathbb{R}$-vector space sheaves, called the sheaf-theoretic differential complex of $\mathcal{A}$.

Moreover, given the validity of the Poincaré Lemma, $\operatorname{Ker}\left(d^{0}\right)=\mathbb{R}$, and the fact that $\mathcal{A}$ is a soft observable algebra sheaf, we obtain:

The sequence of $\mathbb{R}$-vector space sheaves is exact:

$$
\mathbf{0} \rightarrow \mathbb{R} \rightarrow \mathcal{A} \rightarrow \Omega^{1}(\mathcal{A}) \rightarrow \ldots \rightarrow \Omega^{n}(\mathcal{A}) \rightarrow \ldots
$$

Thus, the sheaf-theoretic differential complex of the observable algebra sheaf $\mathcal{A}$ constitutes a resolution of the constant sheaf $\mathbb{R}$ by soft sheaves (which are acyclic with respect to the global sections functor).

The preceding observations elucidate the three basic conceptual issues underlying the generalization of Grassmann's lineal geometric analysis under the prism of sheaf-theoretic localization:

The first pertains to the generalization of Grassmann's exterior algebra from the context of vector spaces defined over a field to the context of vector sheaves defined over a sheaf of algebras of observables.

The second pertains to conceptualizing and expressing Grassmann's scheme algebraically as a process of resolution of the constants, represented by the field of definition, the coordinatizers of points. 
The third pertains to viewing this process of resolution of the constants in terms of the notion of a differential complex, where the latter is based on the action of the exterior derivation operator $d$, characterized by the property that $d^{2}=0$, that is, the coboundary law.

Most important, in cases where the observable algebra sheaf is the sheaf of smooth functions defined on a differential manifold, the above sheaf-theoretic process of resolving the constants provides a clear manifestation of de Rham's cohomological scheme. In precise terms, the sheaf cohomology with coefficients in the constant sheaf $\mathbb{R}$, is isomorphic with the de Rham cohomology of $X$. We emphasize that $\mathbb{R}$ denotes the domain of constants (locally constant sections) of the observable algebra sheaf of smooth functions $\mathcal{A}$ defined on a smooth finite dimensional manifold $X$, identified as the spectrum of $\mathcal{A}$. The same is equally true of the Čech cohomology with values in the constant sheaf $\mathbb{R}$. In short, all the respective cohomology groups are isomorphic:

$$
H^{n}(X ; \mathbb{R}) \cong H_{d R}{ }^{n}(X)
$$

The above isomorphism, first proved by André Weil, establishes the sheaf-theoretic formulation of de Rham's theorem. In order to gain a deeper insight it is of value to describe de Rham's initial motive and follow the type of cohomological unfolding it implements in terms of differential forms.

The essential issue is the disclosure of singular points on a manifold, given that its point structure is only implicitly assumed initially, according to the preceding. This disclosure may be though of in terms of a coboundary operator giving rise to a differential complex, where it is considered as an exterior derivation operator acting on differential forms that obey the rules of Grassmann's lineal geometric analysis. The basic idea is that differential forms are objects which can be temporally integrated over chains in a way that is compatible with pull-back operations. If the result of this integration procedure is not trivial, then the obtained residue characterizes invariantly a singular point encycled by an appropriate chain in terms of periods.

According to de Rham, we consider differential forms of degree $p$, $0 \leq p \leq n$, on a finite dimensional smooth manifold of dimension $n$. These differential forms constitute a real vector space, denoted by $\Omega^{p}$. The exterior differential operator acts on forms $\omega^{p}$, such that $d \omega^{p}$ is a form in $\Omega^{p+1}$, via the linear mapping of real vector spaces

$$
d: \Omega^{p} \rightarrow \Omega^{p+1}
$$


satisfying the condition that $d^{2}=0$.

The notion of the differential complex arising in this manner, called the de Rham complex, encapsulates the idea that the image $d \Omega^{p-1}$ of the real vector space $\Omega^{p-1}$ in $\Omega^{p}$ via $d$, lies in the kernel of the following linear mapping $d: \Omega^{p} \rightarrow \Omega^{p+1}$, denoted by $\mho^{p}$. Then, by means of quotienting the de Rham cohomology groups

$$
H_{d R}^{p}(X):=\mho^{p} / d \Omega^{p-1}
$$

are defined, and they are real vector spaces as well. A form $\omega^{p}$ that belongs to $\mho^{p}$, whereby $d \omega^{p}=0$ is called a closed differential form, whereas a form in $\mho^{p}$ expressed as the differential of a form in $\Omega^{p-1}$ is called an exact differential form. Clearly, every exact form is also closed, but the inverse does not hold. Thus, the de Rham cohomology group of some degree structurally measures the discrepancy from exactness at this degree in terms of closed forms failing to be exact.

At a further stage, we consider that $X$ is subdivided into cells, and let $A_{p}$ be the vector space of $p$-chains. This is a real vector space of linear combinations of $p$-cells with real coefficients. The boundary $\partial_{p}$ of a $p$-cell $\circlearrowright_{p}$ is a $(p-1)$-chain with coefficients \pm 1 . This extends by linearity to a boundary operator

$$
\partial: A_{p} \rightarrow A_{p-1}
$$

such that $\partial^{2}=0$.

Next, we consider the vector space of real linear forms defined on $p$-chains, that is the real vector space $A^{p}$ of $p$-cochains, dual to $A_{p}$. For these, there is associated the coboundary operator

$$
\delta: A^{p} \rightarrow A^{p+1}
$$

satisfying the condition that $\delta^{2}=0$. More precisely, since the coboundary operator $\delta$ acting on cochains is dual to the boundary operator $\partial$ acting on chains, for a cochain $\xi^{p}$ in $A^{p}$

$$
\delta \xi^{p}\left(\rho_{p+1}\right)=\xi^{p}\left(\partial \rho_{p+1}\right)
$$


where $\rho_{p+1}$ is a chain $\in A_{p+1}$. We conclude that the image $d A^{p-1}$ of the vector space $A^{p-1}$ in $A^{p}$ via $\delta$, lies in the kernel of $\delta: A^{p} \rightarrow A^{p+1}$, denoted by $\forall^{p}$. Then, the cohomology groups

$$
\bar{H}^{p}(X):=\forall^{p} / d A^{p-1}
$$

are defined by means of quotienting, and they are real vector spaces.

In this context, de Rham's theorem not only proves the isomorphism:

$$
\bar{H}^{n}(X) \cong H_{d R}{ }^{n}(X) \quad(16)
$$

but additionally, it shows explicitly that the above isomorphism is implemented and realized by the integration of differential forms. In particular, a $p$-cell $\circlearrowright_{p}$ is considered as smooth so as to serve as the domain of integration of a corresponding differential form. For instance, for an 1-differential form $\omega$, the expression $\int_{0} \omega$ stands for the line integral of $\omega$ along the 1-cell $\circlearrowright$. In an analogous manner, the integral $\int_{\circlearrowright_{p}} \omega^{p}$ extends by linearity to all $p$-chains $\rho_{p}$, such that:

$$
\delta \xi^{p}\left(\rho_{p}\right)=\int_{\rho_{p}} \omega^{p}
$$

defines a linear form $\xi^{p}$ identified as a cochain in $A^{p}$. Thus, for every degree $p$ there emerge linear mappings

$$
\Psi: \Omega^{p} \rightarrow A^{p}
$$

such that we have

$$
\Psi\left(d \omega^{p}\right)=\delta \Psi\left(\omega^{p}\right)
$$

culminating into the theorem of George Stokes:

$$
\int_{\rho_{p+1}} d \omega^{p}=\int_{\partial \rho_{p+1}} \omega^{p}=\xi^{p}\left(\partial \rho_{p+1}\right)=\delta \xi^{p}\left(\rho_{p+1}\right)
$$


for all $\rho_{p+1} \in A_{p+1}$. Notice that the linear mappings $\Psi$ map the groups $\mho^{p}$ and $d \Omega^{p-1}$ into the groups $\forall^{p}$ and $d A^{p-1}$ correspondingly. We obtain thereby a linear mapping of the quotient groups:

$$
H_{d R}^{p}(X) \rightarrow \bar{H}^{p}(X)
$$

that de Rham's theorem asserts is an isomorphism.

De Rham's considerations additionally involve the homology groups $\bar{H}_{p}(X)$ defined as the quotient groups:

$$
\bar{H}_{p}(X):=\forall_{p} / \partial A_{p+1}
$$

where $\forall_{p}$ is the kernel of $\partial: A_{p} \rightarrow A_{p-1}$. These chains $\rho_{p}$ in $A_{p}$ satisfying the condition $\partial \rho_{p}=0$ are called cycles, whereas those satisfying the condition $\rho_{p}=\partial \rho_{p+1}$ are called boundaries. In this manner, an element of the homology group $\bar{H}_{p}(X)$ denotes an equivalence class of $p$-cycles differing by a boundary. The connotation of a cycle is tantamount to a closed region of integration.

Consider a cochain $\xi^{p}$ in $\forall^{p}$, i.e. $\delta \xi^{p}=0$, called a $p$-cocycle. Then we obtain that

$$
\delta \xi^{p}\left(\rho_{p+1}\right)=\xi^{p}\left(\partial \rho_{p+1}\right)=0
$$

meaning that $\xi^{p}$ is 0 on $\partial A_{p+1}$. In this way, we obtain an $\mathbb{R}$-valued linear form on $\bar{H}_{p}(X)$ that is identically 0 if $\xi^{p}$ is a $p$-coboundary. In the inverse direction, all $\mathbb{R}$-valued linear forms on $\bar{H}_{p}(X)$ are produced likewise, meaning that

$$
\bar{H}^{p}(X)=\operatorname{Hom}\left(\bar{H}_{p}(X), \mathbb{R}\right)
$$

In other words, $\bar{H}^{p}(X)$ is identified as the dual to $\bar{H}_{p}(X)$, i.e. the vector space of all $\mathbb{R}$-valued linear forms on $\bar{H}_{p}(X)$. Hence, the linear mappings $\Psi$ can viewed equivalently as:

$$
\Psi: H_{d R}^{p}(X) \rightarrow \operatorname{Hom}\left(\bar{H}_{p}(X), \mathbb{R}\right)
$$


which maps a closed differential form $\omega^{p}$ to an $\mathbb{R}$-valued linear form defined on the $p$-cycles, and is 0 on the $p$-boundaries. According to de Rham's theorem $\Psi$ is an isomorphism, and the $\mathbb{R}$-values of this linear form are called the periods of the differential form $\omega^{p}$. The injectivity of $\Psi$ means that if all periods of the closed differential form $\omega^{p}$ are 0 , then $\omega^{p}$ is an exact form, i.e. it is the differential of a form $\chi^{p-1}$, equivalently $\omega^{p}=d \chi^{p-1}$. The surjectivity of $\Psi$ means that given periods $\lambda \in \operatorname{Hom}\left(\bar{H}_{p}(X), \mathbb{R}\right)$, then there always exists a closed differential form $\omega^{p}$, that it is associated with these periods.

Again the essential issue of de Rham cohomological analysis is the disclosure of singular points on a manifold in terms of invariant quantities obtained by the integration of differential forms. More precisely, closed differential forms are the natural integrands over cycles, i.e. they can be temporally integrated over closed chains encircling a singular point, such that the result of this integration procedure leaves a residue characterizing this singular point invariantly in terms of periods.

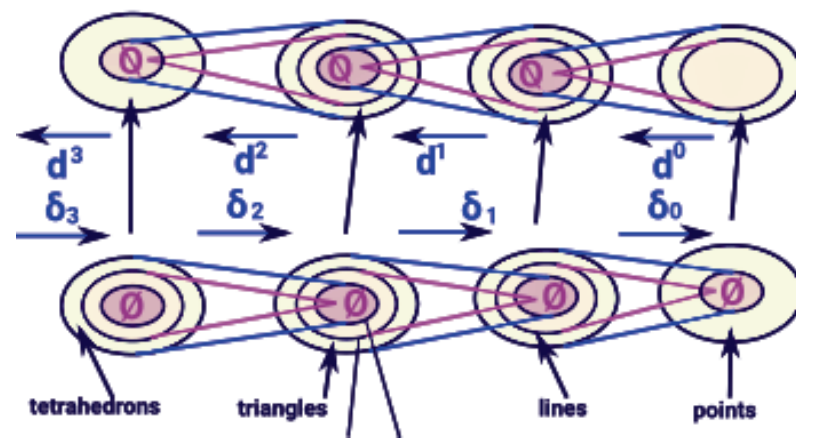

From the dual homology-theoretic viewpoint, the Abelian group $\bar{H}_{0}(X)$ measures the number of connected components of $X$. In turn, the Abelian group $\bar{H}_{1}(X)$ measures how many independent cycles, or 1 dimensional holes $X$ has, modulo the bounding cycles, meaning the boundaries. Since the cohomology groups are dual to their respective homology groups we have the following: The Abelian group $H_{d R}{ }^{0}(X) \cong \bar{H}^{0}(X)$ measures the connected components of $X$ in terms of real valued functions that are locally constant over these components. This is reflected on the dimension of the real vector space $\bar{H}^{0}(X)$. The Abelian group $H_{d R}{ }^{1}(X) \cong \bar{H}^{1}(X)$ is constituted by closed differential 1- 
forms that can be integrated over 1 -cycles enclosing 1-dimensional holes or singular points, allowing them to be characterized in terms of periods.

The most characteristic example is provided by the angular differential 1 -form $\omega$, which is a closed form but not exact, since it cannot be expressed as an exact differential $\omega=d \theta$, where $\theta$ denotes the angle in polar coordinates of a 2 -dimensional Euclidean space. Note that the angle is defined modulo $2 \kappa \pi$, where $\kappa \in \mathbb{Z}$. In cartesian coordinates the angular differential 1-form $\omega$ is expressed as follows:

$$
\omega=\frac{1}{2 \pi} \frac{x d y-y d x}{x^{2}+y^{2}}
$$

It is defined everywhere on $\mathbb{R}^{2}$ except the point $(0,0)$, which is thus a singular point. In this sense, the integration of $\omega$ over oriented 1-cycles encircling the singularity $(0,0)$, i.e. the line integral of the closed differential form $\omega$ :

$$
\int_{\gamma}(27)
$$

over any closed curve $\gamma$ defined on $\mathbb{R}^{2}-(0,0)$ measures its period or winding number around the singular point $(0,0)$, thus disclosing this singular point invariantly.

It is instructive to remember that differential forms $\omega^{p}$ constitute the natural entities to be integrated over cycles of the same dimension. Moreover, if we consider two forms $\omega^{p}$ in $\Omega^{p}$, and $\phi^{(n-p)}$ in $\Omega^{n-p}$, then their exterior product $\omega^{p} \wedge \phi^{(n-p)}$ is a form in $\Omega^{n}$. This differential form can be integrated over $X$, considered as a closed, connected and orientable smooth manifold of finite dimension $n$ :

$$
\int_{X} \omega^{p} \wedge \phi^{(n-p)}
$$

giving rise to a bilinear pairing:

$$
\Omega^{p} \times \Omega^{n-p} \rightarrow \mathbb{R}
$$

under which $\Omega^{n}$ and $\Omega^{n-p}$ become dual spaces. In turn, the above provides an equivalent expression of Poincarés duality, 


$$
\bar{H}^{p}(X) \cong \bar{H}_{n-p}(X) \quad(30)
$$

We close this circle of ideas related to de Rham cohomology by revisiting the sheaf-theoretic form of de Rham's theorem. We recall that the Čech cohomology with values in the constant sheaf $\mathbb{R}$, where $\mathbb{R}$ is the domain of constants (locally constant sections) of the observable algebra sheaf of smooth functions $\mathcal{A}$ on a smooth finite dimensional manifold $X$, conceived as the spectrum of $\mathcal{A}$, is isomorphic with the de Rham cohomology of $X$. This led to the conclusion that all the respective cohomology groups are isomorphic:

$$
H^{n}(X ; \mathbb{R}) \cong H_{d R}^{n}(X)
$$

We recall that the $0-t h$ abelian cohomology group $H^{0}(X ; \mathbb{R}, \mathbb{U})$ emanates purely from 0 -cocycles, i.e. from cochains $\phi:=\left\{f_{\kappa}\right\}$, where $\kappa$ contains only one element, and each $f_{\kappa}$ is a locally constant, real-valued function. We have:

$$
\delta \phi=0 \Leftrightarrow f_{a}=f_{b}
$$

on the intersection $U_{a} \cap U_{b}$ of the open covers $U_{a}$ and $U_{b}$. Therefore, $H^{0}(X ; \mathbb{R}, \mathbb{U})$ is the Abelian group of locally constant real-valued functions. Thus, $H^{0}(X ; \mathbb{R}, \mathbb{U})$ detects the connected components of $X$. More concretely, the dimension of the real vector space $H^{0}(X ; \mathbb{R}, \mathbb{U})$ provides the number of connected components of $X$. If $X$ is connected then $H^{0}(X ; \mathbb{R}, \mathbb{U})=\mathbb{R}$. In this case, $H^{0}(X ; \mathbb{R}, \mathbb{U})$ is the Abelian group of all globally defined constant functions.

For simplicity, we may consider an open cover $V$ of an $n$ dimensional Euclidean space $\mathbb{R}^{n}$ and recall how we obtain $H^{0}(V ; \mathbb{R})$. Then, the locally constant real-valued smooth functions in $\mathbb{R}^{n}$ over $V$ constitute the Abelian group $H^{0}(V ; \mathbb{R})$. These locally constant realvalued smooth functions $f$ over $V$ are exactly the solutions of the differential equation:

$$
d f=0 \Leftrightarrow \sum_{i} \frac{\partial f}{\partial x_{i}} d x^{i}=0
$$


Generalizing, for any smooth manifold the Abelian group $H^{0}(V ; \mathbb{R})$, where $V$ is an open cover, may be interpreted as the real vector space of local solutions on $V$ of the differential equation $d f=0$, where $f$ is qualified accordingly as a locally constant real-valued smooth function over $V$.

Most important, since $H^{n}(X ; \mathbb{R}) \cong H_{d R}{ }^{n}(X)$ at all orders, the constant sheaf of the reals $\mathbb{R}$, in its role as an Abelian coefficient sheaf of cohomology, should furnish the description of all higher cohomology groups in a way analogous to the above case. In this manner, $H^{1}(X ; \mathbb{R})$ can be viewed as the real vector space of solutions of the differential equation $d \omega=0$, where $\omega$ is a closed differential 1 -form in $\Omega^{1}$, modulo the exact differential forms, by which we mean these solutions $\omega=d \sigma$, where $\sigma$ in $\Omega^{0}$, considered trivial due to the validity of the coboundary law. Note that the difference between closed forms and exact forms is a global feature of $X$ reflecting the topological complexity of singular points of $X$.

If $X$ is a smooth manifold, we may express $\omega$ in the form $\omega=\sum_{i} \omega_{i} d x^{i}$, where the coordinate functions $\omega_{i}$ are smooth functions. Recalling that $\omega$ constitutes the natural entity to be integrated over cycles of the same dimension, we consider $\omega$ as a function on cycles:

$$
o \mapsto f_{o} \omega
$$

The important thing is that since the evaluation of this integral gives the period of $\omega$ with respect to an integration cycle $o$, the above function is a locally constant real-valued function on homologous cycles.

Conversely, considering a general differential 1 -form $\omega$, we think of it as a function on smooth paths $\gamma$ :

$$
\gamma \mapsto \int_{\gamma} \omega
$$

and examine when the above function is a locally constant real-valued function on $X$. In other words, we are interested in those $\omega$ for which the line integral $\int_{\gamma} \omega$ remains constant under perturbations of $\gamma$, while keeping its endpoints fixed. This happens only if $\omega$ is a closed differential 1 -form, i.e. $d \omega=0$, meaning that the required condition is fulfilled by the solutions of the differential equation $d \omega=0$ modulo the 
solutions $\omega=d \sigma$, where $\sigma$ in $\Omega^{0}$, satisfying trivially the local constancy condition.

Thus, $H^{1}(X ; \mathbb{R})$ is a real vector space determined by the real-valued locally constant integrals of closed differential 1 -forms modulo those that are trivially locally constant of exact differential 1-forms. The same prescription can be generalized to all higher order cohomology groups by the replacement of line integrals by their higher dimensional analogues. In conclusion, we realize that the constant Abelian sheaf of the reals $\mathbb{R}$ determines by the localization of the notion of constancy to local constancy all de Rham cohomology groups in its function as a coefficient sheaf of Čech cohomology. 
